\title{
THE INFLUENCE OF DOMAIN TRUNCATION ON THE PERFORMANCE OF OPTIMIZED SCHWARZ METHODS*
}

\author{
YINGXIANG XU ${ }^{\dagger}$
}

\begin{abstract}
Optimized Schwarz methods enhance convergence using optimized transmission conditions between subdomains. The optimization is usually performed for a model problem on an unbounded domain and two subdomains represented by half spaces. The influence of the domain decomposition geometry on the convergence and the optimized parameters is thus lost in the process, and it is not even theoretically clear if the results published for the unbounded domain still hold in concrete applications where the domains are bounded. We prove here rigorously for a two-subdomain decomposition that the asymptotic performance of optimized Schwarz methods derived from an unbounded domain analysis still holds in the case of a bounded domain, but the constants in the best choice of parameters and convergence rate estimates are influenced by the domain truncation. We obtain accurate estimates for this influence and show theoretically that the domain truncation has more remarkable influence for the slowly converging optimized Schwarz methods than for those converging fast. When the subdomain size is very small, our new optimized parameters lead to much faster algorithms than those obtained from an unbounded domain analysis. We illustrate our theoretical results with numerical experiments.
\end{abstract}

Key words. optimized Schwarz methods, domain decomposition methods, transmission conditions, influence of domain truncation

AMS subject classifications. 65N55, 65F10

1. Introduction. In the field of high performance computing, domain decomposition (DD) methods are among the most flexible and efficient techniques for the simulation of large scale problems; see for example the monograph [42]. DD methods can deal naturally with complicated heterogeneous problems and reduce dramatically their complexity, leading to scalable solvers and preconditioners. Beyond the famous finite element tearing and interconnecting (FETI) methods invented by Farhat and Roux [13] and the balancing domain decomposition by constraints (BDDC) algorithm introduced by Dohrmann [8], the optimized Schwarz methods were developed based on a nonoverlapping variant of the alternating Schwarz method proposed by Lions [31]. They can be used with and without overlap. Their performance can be optimized by appropriately choosing parameters in the transmission conditions between subdomains, and the methods have received considerable attention over the past decade in the DD literature; for overviews, see [14, 35] and the monograph [10]. Optimized Schwarz methods have not only led to many theoretical developments, for example [17, 20, 22, 23, 29, 32, 33], but also have been proven very useful in many applications: for instance, we refer to $[3,7,19,24]$ for Helmholtz problems, [4, 16, 34, 41] for advection diffusion problems, [1, 9, 11, 36, 37] for Maxwell's equations, [39, 40] for shallow water problems, [2] for primitive equations, [27] for the fluid-structure interaction problem, and [28] for an electrocardiology simulation. Optimized Schwarz methods can be used as efficient preconditioners as well; for example, see $[18,30]$ for an optimized Schwarz preconditioner. In addition, optimized Schwarz methods contribute also to the development of many innovative preconditioners, including the sweeping preconditioner that recently has received much attention (see [12, 38, 43, 44, 45]) and the source transfer method (see [6]). We refer the reader to [5] for the precise relation of those methods.

${ }^{*}$ Received August 23, 2017. Accepted July 9, 2018. Published online on October 12, 2018. Recommended by Daniel B. Szyld. The research is supported by NSFC-11671047,11471047 and the Fundamental Research Funds for the Central Universities (No. 2412018ZD001).

${ }^{\dagger}$ School of Mathematics and Statistics, Northeast Normal University, Changchun 130024, China (yxxu@nenu.edu.cn). 


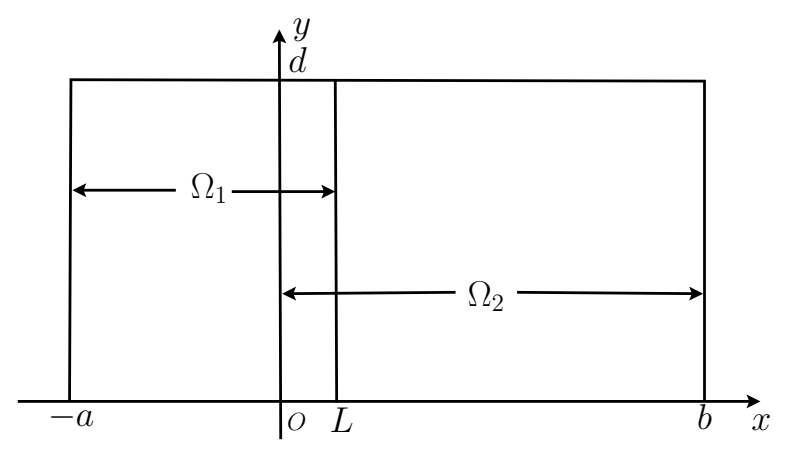

FIG. 1.1. The domain decomposition used.

The optimization of transmission conditions in optimized Schwarz methods is generally based on Fourier techniques applied to a model problem with a decomposition into infinite domains. However, in applications, the computational domain is finite, and the decomposition is often given according to physical properties of the underlying model or by a mesh partitioner, and thus, in many applications, subdomains are of various shapes and sizes. The influence of the subdomain geometry on the convergence and the optimized parameters in optimized Schwarz methods is not yet well understood. A first step in this direction can be found in [15], where it was shown that the subdomain geometry and the boundary conditions indeed affect the performance of the optimized Schwarz method with Robin transmission conditions. A further investigation was performed in [21], where the influence of the subdomain geometry on optimized Schwarz methods with two-sided transmission conditions was addressed in detail. We note here that in certain cases the interface geometry can correspond to model problems with variable coefficients; see [22] for a corresponding analysis of optimized Schwarz methods using the technique of separation of variables.

We study here in detail the influence of the subdomain geometry on optimized Schwarz methods for the model problem

$$
\Delta u-\eta u=f \quad \text { in } \Omega,\left.\quad u\right|_{\partial \Omega}=0,
$$

where $\eta \geq 0$ is a model parameter, $\Omega=\{(x, y) \mid-a<x<b, 0<y<d\}$ is decomposed into two subdomains $\Omega=\Omega_{1} \cup \Omega_{2}$ with $\Omega_{1}=\{(x, y) \mid-a<x<L, 0<y<d\}$, $\Omega_{2}=\{(x, y) \mid 0<x<b, 0<y<d\}, L \geq 0$ the overlap between the subdomains, and $a, b, d$ positive domain parameters. Without loss of generality, we assume $a+L<b$; see Figure 1.1. This setting contains many existing results as special cases, e.g., $a=b=\infty$ leads to the case of an infinite domain decomposition analyzed in [14]; $a=b$ finite leads to the finite symmetric domain decomposition in [15], and if $b=\infty$ and $a$ is finite, then we have a one-sided bounded domain decomposition, which is similar to a circular domain decomposition; see [20, 23]. In this paper, we would like to consider only the two-subdomain decomposition described above, which allows us to obtain asymptotically accurate formulas of the best transmission parameters. When the physical domain is decomposed into arbitrary number of subdomains (cross points may be present), we refer the reader to [25] for a convergence analysis of the synchronous optimized Schwarz method with Robin transmission condition and to [26] for the asynchronous case. Beyond the continuous level analysis, see [18] for an algebraic analysis for problems defined on irregular physical domains. We remark here that for the case of multiple subdomains, the expression of the convergence factor is too complicated to derive explicit formulas for the optimal transmission parameters by the optimization technique. 


\section{ETNA}

Kent State University and

Johann Radon Institute (RICAM)

A parallel Schwarz method for problem (1.1) is defined by solving the following problems until convergence, for $n=1,2, \ldots$,

$$
\begin{aligned}
& \Delta u_{1}^{n}-\eta u_{1}^{n}=f, \quad \text { in } \Omega_{1}, \quad \Delta u_{2}^{n}-\eta u_{2}^{n}=f, \quad \text { in } \Omega_{2}, \\
& u_{1}^{n}(-a, y)=0, \quad 0<y<d, \quad u_{2}^{n}(b, y)=0, \quad 0<y<d \text {, }
\end{aligned}
$$

with the transmission conditions

$$
\mathcal{B}_{1} u_{1}^{n}(L, \cdot)=\mathcal{B}_{1} u_{2}^{n-1}(L, \cdot), \quad \mathcal{B}_{2} u_{2}^{n}(0, \cdot)=\mathcal{B}_{2} u_{1}^{n-1}(0, \cdot),
$$

where $u_{2}^{0}(L, \cdot)$ and $u_{1}^{0}(0, \cdot)$ are given initial guesses.

Choosing $\mathcal{B}_{i}=I, \mathcal{B}_{i}=\partial_{n}+p, \mathcal{B}_{i}=\partial_{n}+p-q \partial_{y y}$, or $\mathcal{B}_{i}=\partial_{n}+p_{i}$ will result in Dirichlet, Robin, second-order, or two-sided Robin transmission conditions; see [14]. We will optimize the transmission parameters and analyze the corresponding performance of the above mentioned transmission conditions under the given domain decomposition setting and compare them to those obtained from the infinite domain decomposition analysis.

2. The classical Schwarz algorithm. For Fourier analysis, we assume that the subdomain solutions $u_{i}^{n}(x, y), i=1,2$, have the form

$$
u_{i}^{n}(x, y)=\sum_{k=1}^{\infty} \hat{u}_{i}^{n}(x, k) \sin (k y) .
$$

Note here, in practical computations, the Fourier frequency $k$ lies in between $k_{\min }$ and $k_{\max }$, the lowest and highest frequencies involved in the computation. For a homogeneous Dirichlet boundary condition, if a uniform mesh is applied, $h$ is the mesh size along the interface, and the interface length is $d$, then one can estimate $k_{\min }=\pi / d$ and $k_{\max }=\pi / h$; see [14].

Inserting (2.1) into (1.2), we obtain after a short calculation assuming $f=0$, which represents the error equations, that

$$
\begin{aligned}
\partial_{x x} \hat{u}_{1}^{n}-\left(\eta+k^{2}\right) \hat{u}_{1}^{n} & =0, & \partial_{x x} \hat{u}_{2}^{n}-\left(\eta+k^{2}\right) \hat{u}_{2}^{n} & =0, \\
\hat{u}_{1}^{n}(-a, k) & =0, & \hat{u}_{2}^{n}(b, k) & =0 .
\end{aligned}
$$

The closed form solutions of (2.2) are

$$
\begin{aligned}
& \hat{u}_{1}^{n}(x, k)=A_{1}^{n}(k) \sinh \left(\sqrt{\eta+k^{2}}(x+a)\right), \\
& \hat{u}_{2}^{n}(x, k)=A_{2}^{n}(k) \sinh \left(\sqrt{\eta+k^{2}}(x-b)\right) .
\end{aligned}
$$

For the classical Schwarz method we have $\mathcal{B}_{i}=I$, i.e., the identity operator, and we obtain using (1.3)

$$
\hat{u}_{1}^{n}(L, k)=\hat{u}_{2}^{n-1}(L, k), \quad \hat{u}_{2}^{n}(0, k)=\hat{u}_{1}^{n-1}(0, k) \quad \text { for all } k \in \mathbb{K}:=\left[k_{\min }, k_{\max }\right] .
$$

Inserting (2.3) evaluated at $x=0$ and $x=L$, respectively, into (2.4) leads to a recurrence for the constants

$$
A_{1}^{n}(k)=\frac{\sinh \left(\sqrt{\eta+k^{2}}(L-b)\right)}{\sinh \left(\sqrt{\eta+k^{2}}(a+L)\right)} A_{2}^{n-1}(k), \quad A_{2}^{n}(k)=\frac{\sinh \left(\sqrt{\eta+k^{2}} a\right)}{\sinh \left(\sqrt{\eta+k^{2}}(-b)\right)} A_{1}^{n-1}(k) .
$$

We therefore get

$$
A_{1}^{2 n}(k)=\rho_{c l a}^{n} A_{1}^{0}(k), \quad A_{2}^{2 n}(k)=\rho_{c l a}^{n} A_{2}^{0}(k)
$$


with the contraction factor

$$
\rho_{c l a}(k, L, \eta, a, b):=\frac{\sinh \left(\sqrt{\eta+k^{2}}(b-L)\right)}{\sinh \left(\sqrt{\eta+k^{2}}(a+L)\right)} \frac{\sinh \left(\sqrt{\eta+k^{2}} a\right)}{\sinh \left(\sqrt{\eta+k^{2}} b\right)} .
$$

To understand the influence of the geometry on the classical Schwarz method, we need to investigate the properties of the contraction factor $\rho_{c l a}$. We need the following lemmas, which also will be useful throughout the rest of the paper.

LEMMA 2.1. The function $f(x)=x \operatorname{coth}(x)$ is positive and monotonically increasing in $x$, for $x>0$, and $\lim _{x \rightarrow+\infty} x \operatorname{coth}(x)=+\infty$.

Proof. Let $g(x):=e^{2 x}-e^{-2 x}-4 x$. The derivative of $g$ is $g^{\prime}(x)=2\left(e^{2 x}+e^{-2 x}\right)-4>0$ for $x>0$, which means, together with $g(0)=0$, that the function $g$ is positive for $x>0$. Therefore, the derivative $f^{\prime}(x)=\operatorname{coth}(x)+x\left(1-\operatorname{coth}^{2}(x)\right)=\frac{g(x)}{\left(e^{x}-e^{-x}\right)^{2}}>0$ for $x>0$. Together with the fact that $\lim _{x \rightarrow 0} f(x)=1$, we have that $f(x)$ is positive and monotonically increasing in $x$, for $x>0$, and clearly the limit as $x \rightarrow+\infty$ is infinity.

LEMMA 2.2. Let $G(k, \eta, a, b):=\frac{\sqrt{\eta+k^{2}}}{4}\left(\operatorname{coth}\left(\sqrt{\eta+k^{2}} a\right)+\operatorname{coth}\left(\sqrt{\eta+k^{2}} b\right)\right)$. Then $G(k, \eta, a, b)$ increases monotonically in $k$ for $k>0$ and $\eta, a, b$ fixed. The function $G(k, \eta, a, b)$ can also be rewritten as $G(k, \eta, a, b)=\frac{\sqrt{\eta+k^{2}}}{2} \frac{1-e^{-2 \sqrt{\eta+k^{2}}}(a+b)}{\left(1-e^{-2 \sqrt{\eta+k^{2}} a}\right)\left(1-e^{-2 \sqrt{\eta+k^{2}} b}\right)}$.

Proof. By Lemma 2.1, the function $G(k, \eta, a, b)$ is the sum of two increasing functions with positive weights, which implies a monotonic growth in $k$. The second assertion is obtained by a direct calculation.

THEOREM 2.3. With overlap $L>0$, the classical Schwarz method has asymptotically, as $L$ tends to 0 , the convergence factor

$$
\max _{k \in \mathbb{K}}\left|\rho_{c l a}(k, L, \eta, a, b)\right|=1-4 G_{\min } L+O\left(L^{2}\right),
$$

where $G_{\min }=G\left(k_{\min }, \eta, a, b\right)$. If there is no overlap, then the classical Schwarz method does not converge.

Proof. Let $f(x):=\frac{\sinh (r x)}{\sinh (s x)}$ with $0<r<s$. Then, when $x>0$ we have that

$$
f^{\prime}(x)=(\cosh (r x) \sinh (s x) r-\sinh (r x) \cosh (s x) s) / \sinh ^{2}(s x)<0
$$

since for $x>0$ the denominator is greater than 0 and the numerator is equivalent to $\frac{\sinh (r x) \sinh (s x)}{x}(\operatorname{coth}(r x) r x-\operatorname{coth}(s x) s x)$ and thus less than 0 by Lemma 2.1. Therefore, both $\frac{\sinh \left(\sqrt{\eta+k^{2}}(b-L)\right)}{\sinh \left(\sqrt{\eta+k^{2}} b\right)}$ and $\frac{\sinh \left(\sqrt{\eta+k^{2}} a\right)}{\sinh \left(\sqrt{\eta+k^{2}}(a+L)\right)}$ decrease monotonically in $k$ for $k>0$ and as the product of these two positive functions, $\rho_{c l a}$ is decreasing monotonically in $k$ for $k>0$. As a consequence, $\rho_{c l a}$ attains its maximum at $k=k_{\min }$. A Taylor expansion of the contraction factor with respect to $L$ for $L$ small at $k_{\min }$ gives (2.5). The second assertion follows from the fact that $\rho_{c l a}=1$ for any $k$ when $L=0$.

3. The optimized transmission conditions. The classical Schwarz method has many drawbacks: it converges very slow in the overlapping case and does not converge in the nonoverlapping case. To improve the performance of the Schwarz algorithm (1.2), we can use Robin transmission conditions. We start with $\mathcal{B}_{i}=\frac{\partial}{\partial n_{i}}+\mathcal{S}_{i}, i=1,2$, and obtain

$$
\begin{aligned}
\frac{\partial}{\partial n_{1}} \hat{u}_{1}^{n}+\sigma_{1}(k) \hat{u}_{1}^{n}=\frac{\partial}{\partial n_{1}} \hat{u}_{2}^{n-1}+\sigma_{1}(k) \hat{u}_{2}^{n-1}, & \text { at } x=L, \\
\frac{\partial}{\partial n_{2}} \hat{u}_{2}^{n}+\sigma_{2}(k) \hat{u}_{2}^{n}=\frac{\partial}{\partial n_{2}} \hat{u}_{1}^{n-1}+\sigma_{2}(k) \hat{u}_{1}^{n-1}, & \text { at } x=0,
\end{aligned}
$$


where $\sigma_{i}, i=1,2$, are the Fourier symbols of $\mathcal{S}_{i}$. Inserting (2.3) into (3.1) and (3.2), we obtain

and

$$
A_{1}^{n}(k)=\left.\frac{\left(\frac{\partial}{\partial x}+\sigma_{1}(k)\right) \sinh \left(\sqrt{\eta+k^{2}}(x-b)\right)}{\left(\frac{\partial}{\partial x}+\sigma_{1}(k)\right) \sinh \left(\sqrt{\eta+k^{2}}(x+a)\right)}\right|_{x=L} A_{2}^{n-1}(k)
$$

$$
A_{2}^{n}(k)=\left.\frac{\left(\frac{\partial}{\partial x}-\sigma_{2}(k)\right) \sinh \left(\sqrt{\eta+k^{2}}(x+a)\right)}{\left(\frac{\partial}{\partial x}-\sigma_{2}(k)\right) \sinh \left(\sqrt{\eta+k^{2}}(x-b)\right)}\right|_{x=0} A_{1}^{n-1}(k) .
$$

Thus, we have

$$
A_{1}^{2 n}(k)=\rho^{n} A_{1}^{0}(k), \quad A_{2}^{2 n}(k)=\rho^{n} A_{2}^{0}(k)
$$

with the contraction factor

$$
\begin{aligned}
\rho(k, L, & \left.\eta, \sigma_{1}(k), \sigma_{2}(k), a, b\right) \\
:= & \frac{\sqrt{\eta+k^{2}} \cosh \left(\sqrt{\eta+k^{2}}(b-L)\right)-\sigma_{1}(k) \sinh \left(\sqrt{\eta+k^{2}}(b-L)\right)}{\sqrt{\eta+k^{2}} \cosh \left(\sqrt{\eta+k^{2}}(a+L)\right)+\sigma_{1}(k) \sinh \left(\sqrt{\eta+k^{2}}(a+L)\right)} \times \\
& \frac{\sqrt{\eta+k^{2}} \cosh \left(\sqrt{\eta+k^{2}} a\right)-\sigma_{2}(k) \sinh \left(\sqrt{\eta+k^{2}} a\right)}{\sqrt{\eta+k^{2}} \cosh \left(\sqrt{\eta+k^{2}} b\right)+\sigma_{2}(k) \sinh \left(\sqrt{\eta+k^{2}} b\right)} .
\end{aligned}
$$

The convergence factor $\rho$ can be made identically zero with the optimal choice

$$
\sigma_{1}(k)=\sqrt{\eta+k^{2}} \operatorname{coth}\left(\sqrt{\eta+k^{2}}(b-L)\right), \quad \sigma_{2}(k)=\sqrt{\eta+k^{2}} \operatorname{coth}\left(\sqrt{\eta+k^{2}} a\right),
$$

which results in the well-known optimal Schwarz algorithm. However, this optimal choice corresponds to a nonlocal transmission condition: it represents a Dirichlet-to-Neumann operator and is thus rather costly. To obtain an optimized Schwarz method, one approximates $\sigma_{i}(k)$ such that the information near the interface is transmitted effectively locally. Because polynomial symbols are local, we search for approximations of the form $\sigma_{i}^{a p p}(k)=p_{i}+$ $q_{i} k^{2}, i=1,2$, with $p_{i}, q_{i}$ constants. The idea of optimized Schwarz methods is to determine the transmission parameters $p_{i}, q_{i}$ by solving a min-max problem under certain constraints,

$$
\min _{p_{i}, q_{i} \geq 0} \max _{k \in \mathbb{K}}\left|\rho\left(k, L, \eta, \sigma_{1}^{a p p}(k), \sigma_{2}^{a p p}(k), a, b\right)\right| .
$$

In addition, since the frequency $k$ is involved in the contraction factor $\rho$ in a complicated fashion, it is in general not possible to solve (3.3) in closed form. To overcome this difficulty, we search for an approximate solution to (3.3) which is asymptotically accurate. To this end, we introduce the following approximation $\rho_{a p p}$ of the convergence factor $\rho$ :

$$
\rho_{\text {app }}\left(k, L, \eta, \sigma_{1}(k), \sigma_{2}(k)\right)=\frac{\sqrt{\eta+k^{2}}-\sigma_{1}(k)}{\sqrt{\eta+k^{2}}+\sigma_{1}(k)} \frac{\sqrt{\eta+k^{2}}-\sigma_{2}(k)}{\sqrt{\eta+k^{2}}+\sigma_{2}(k)} e^{-2 \sqrt{\eta+k^{2}} L} .
$$

When the approximate symbols $\sigma_{i}^{a p p}(k), i=1,2$, are used, the approximate convergence factor $\rho_{\text {app }}$ is precisely the convergence factor from the infinite domain decomposition analysis; see [14].

THEOREM 3.1 (Approximation of the convergence factor). The convergence factor $\rho$ is approximated by $\rho_{\text {app }}$ with the estimate

$$
\begin{aligned}
\mid \rho\left(k, L, \eta, \sigma_{1}(k), \sigma_{2}(k), a, b\right) & -\rho_{a p p}\left(k, L, \eta, \sigma_{1}(k), \sigma_{2}(k)\right) \mid \\
& \leq 2\left(e^{-2 \sqrt{\eta+k^{2}} b}+e^{-2 \sqrt{\eta+k^{2}}(a+L)}\right) .
\end{aligned}
$$




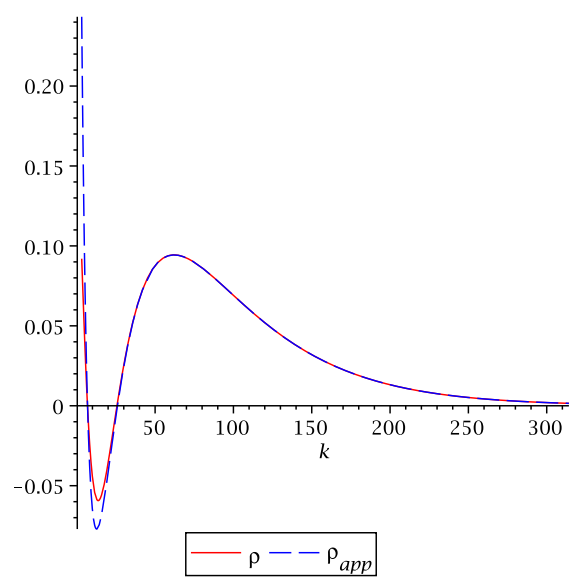

FIG. 3.1. The convergence factor $\rho$ compared to its approximation $\rho_{\text {app }}$ when $\eta=2, a=0.1, b=0.5$, $\sigma_{1}=7, \sigma_{2}=26$, and $L=1 / 100$, for $k \in[\pi, 100 \pi]$.

Proof. Noting that

$\rho=\rho_{a p p}+\left(1-\rho_{a p p}\right)\left(\frac{\sqrt{\eta+k^{2}}-\sigma_{2}(k)}{\sqrt{\eta+k^{2}}+\sigma_{2}(k)} e^{-2 \sqrt{\eta+k^{2}} b}+\frac{\sqrt{\eta+k^{2}}-\sigma_{1}(k)}{\sqrt{\eta+k^{2}}+\sigma_{1}(k)} e^{-2 \sqrt{\eta+k^{2}}(a+L)}\right)$,

we obtain

$$
\begin{aligned}
\mid \rho & -\rho_{a p p} \mid \\
& =\left|\left(1-\rho_{a p p}\right)\left(\frac{\sqrt{\eta+k^{2}}-\sigma_{2}(k)}{\sqrt{\eta+k^{2}}+\sigma_{2}(k)} e^{-2 \sqrt{\eta+k^{2}} b}+\frac{\sqrt{\eta+k^{2}}-\sigma_{1}(k)}{\sqrt{\eta+k^{2}}+\sigma_{1}(k)} e^{-2 \sqrt{\eta+k^{2}}(a+L)}\right)\right| \\
& \leq\left|1-\rho_{a p p}\right|\left(\left|\frac{\sqrt{\eta+k^{2}}-\sigma_{2}(k)}{\sqrt{\eta+k^{2}}+\sigma_{2}(k)} e^{-2 \sqrt{\eta+k^{2}} b}\right|+\left|\frac{\sqrt{\eta+k^{2}}}{\sqrt{\eta+k^{2}}+\sigma_{1}(k)} e_{1}^{-2 \sqrt{\eta+k^{2}}(a+L)}\right|\right) \\
& \leq 2\left(e^{-2 \sqrt{\eta+k^{2}} b}+e^{-2 \sqrt{\eta+k^{2}}(a+L)}\right),
\end{aligned}
$$

where $\sigma_{i}(k)>0$ and $-1 \leq \rho_{a p p} \leq 1$ were used.

Theorem 3.1 shows that $\rho_{\text {app }}$ approximates the convergence factor $\rho$ very well if the frequency $k$ is large. However, if the frequency $k$ is small, then the difference between the exact convergence factor and its approximation can be quite large; see Figure 3.1. As a consequence, directly optimizing the approximate convergence factor $\rho_{a p p}$ can not generate the best transmission parameters for our domain decomposition setting, especially when the subdomain size parameters $a$ and/or $b$ are small. We will discuss this issue in more detail in Section 4.

3.1. A low frequency approximation. From the asymptotic behavior of the classical Schwarz method described in Theorem 2.3, we see that the method is inefficient in damping low frequency components of the error. To overcome this, one can expand the optimal symbols $\sigma_{i}(k)$ in a Taylor series about $k=0$ to obtain a local transmission condition which is efficient in damping low frequencies. By truncating the Taylor expansion at zeroth order, we obtain the Taylor transmission condition of order 0 (T0)

$$
p_{1}^{T 0}=\sqrt{\eta} \operatorname{coth}(\sqrt{\eta}(b-L)), \quad p_{2}^{T 0}=\sqrt{\eta} \operatorname{coth}(\sqrt{\eta} a), \quad q_{1}^{T 0}=q_{2}^{T 0}=0 .
$$


The corresponding convergence factor is

$$
\begin{aligned}
\rho_{T 0}( & \left.k, L, \eta, p_{1}^{T 0}, p_{2}^{T 0}, a, b\right) \\
= & \frac{\sqrt{\eta+k^{2}} \cosh \left(\sqrt{\eta+k^{2}}(b-L)\right)-\sqrt{\eta} \operatorname{coth}(\sqrt{\eta}(b-L)) \sinh \left(\sqrt{\eta+k^{2}}(b-L)\right)}{\sqrt{\eta+k^{2}} \cosh \left(\sqrt{\eta+k^{2}}(a+L)\right)+\sqrt{\eta} \operatorname{coth}(\sqrt{\eta}(b-L)) \sinh \left(\sqrt{\eta+k^{2}}(a+L)\right)} \times \\
& \frac{\sqrt{\eta+k^{2}} \cosh \left(\sqrt{\eta+k^{2}} a\right)-\sqrt{\eta} \operatorname{coth}(\sqrt{\eta} a) \sinh \left(\sqrt{\eta+k^{2}} a\right)}{\sqrt{\eta+k^{2}} \cosh \left(\sqrt{\eta+k^{2}} b\right)+\sqrt{\eta} \operatorname{coth}(\sqrt{\eta} a) \sinh \left(\sqrt{\eta+k^{2}} b\right)} .
\end{aligned}
$$

Before analyzing the performance of the corresponding optimized Schwarz method (1.2), we introduce first the following Lemma, which is also useful for the rest of the paper.

LEMMA 3.2. For any $0 \leq L<b-a$, let

$$
\begin{aligned}
\rho_{b}\left(k, L, \eta, p_{1}, p_{2}\right) & :=\frac{\sqrt{\eta+k^{2}} \cosh \left(\sqrt{\eta+k^{2}}(b-L)\right)-p_{1} \sinh \left(\sqrt{\eta+k^{2}}(b-L)\right)}{\sqrt{\eta+k^{2}} \cosh \left(\sqrt{\eta+k^{2}} b\right)+p_{2} \sinh \left(\sqrt{\eta+k^{2}} b\right)} \\
\rho_{a}\left(k, L, \eta, p_{1}, p_{2}\right) & :=\frac{\sqrt{\eta+k^{2}} \cosh \left(\sqrt{\eta+k^{2}} a\right)-p_{2} \sinh \left(\sqrt{\eta+k^{2}} a\right)}{\sqrt{\eta+k^{2}} \cosh \left(\sqrt{\eta+k^{2}}(a+L)\right)+p_{1} \sinh \left(\sqrt{\eta+k^{2}}(a+L)\right)},
\end{aligned}
$$

and let $k_{b}\left(L, p_{1}\right)>0$ be such that

$$
\rho_{b}\left(k_{b}, L, \eta, p_{1}, p_{2}\right)=0 \quad \text { when } \quad p_{1}>\sqrt{\eta+k_{\min }^{2}} \operatorname{coth}\left(\sqrt{\eta+k_{\min }^{2}}(b-L)\right)
$$

and $k_{a}\left(L, p_{2}\right)>0$ such that

$$
\rho_{a}\left(k_{a}, L, \eta, p_{1}, p_{2}\right)=0 \quad \text { when } \quad p_{2}>\sqrt{\eta+k_{\min }^{2}} \operatorname{coth}\left(\sqrt{\eta+k_{\min }^{2}} a\right) .
$$

Then,

a) $k_{b}\left(L, p_{1}\right)$ (resp. $\left.k_{a}\left(L, p_{2}\right)\right)$ behaves asymptotically as $p_{1}\left(\right.$ resp. $\left.p_{2}\right)$ for $p_{1} \rightarrow+\infty$ (resp. $\left.p_{2} \rightarrow+\infty\right)$;

b) If $p_{1}=p_{2}=p$, then $k_{b}(L, p)>k_{a}(L, p)$ for sufficiently large $p$;

c) $\rho_{b}\left(k, L, \eta, p_{1}, p_{2}\right)$ (resp. $\rho_{a}\left(k, L, \eta, p_{1}, p_{2}\right)$ ) is negative and increasing in $k$ for $k \in\left(k_{\min }, k_{b}\left(L, p_{1}\right)\right)$ (resp. $\left.k \in\left(k_{\min }, k_{a}\left(L, p_{2}\right)\right)\right)$;

d) $\rho_{b}\left(k, L, \eta, p_{1}, p_{2}\right)$ (resp. $\left.\rho_{a}\left(k, L, \eta, p_{1}, p_{2}\right)\right)$ is positive and asymptotically increasing in $k$ for $0<k<c L^{-\frac{1}{2}}$ and $L$ small enough if

$$
\begin{aligned}
p_{1} & <\sqrt{\eta+k_{\min }^{2}} \operatorname{coth}\left(\sqrt{\eta+k_{\min }^{2}}(b-L)\right) \\
\left(\text { respectively, } p_{2}\right. & \left.<\sqrt{\eta+k_{\min }^{2}} \operatorname{coth}\left(\sqrt{\eta+k_{\min }^{2}} a\right)\right) .
\end{aligned}
$$

Proof.

a) We only prove the result for $k_{b}\left(L, p_{1}\right)$. Note that

$$
p_{1}=\sqrt{\eta+k_{b}^{2}\left(L, p_{1}\right)} \operatorname{coth}\left(\sqrt{\eta+k_{b}^{2}\left(L, p_{1}\right)}(b-L)\right) \approx k_{b}\left(L, p_{1}\right)
$$

since $\operatorname{coth}(x) \rightarrow 1$ converges faster than any polynomial as $x \rightarrow+\infty$, and we obtain the assertion independently of $L \geq 0$ for $p_{1}$ large enough. 
b) We prove this by contradiction. If $k_{b}=k_{a}$, then we have for $p_{1}=p_{2}=p$ that $\operatorname{coth}\left(\sqrt{\eta+k_{b}^{2}(L, p)}(b-L)\right)=\operatorname{coth}\left(\sqrt{\eta+k_{b}^{2}(L, p)} a\right)$, which contradicts the assumption $a+L<b$. From assertion a) and the fact that $a+L<b$, we have for $p$ large enough that $k_{b}(L, p)(b-L)>k_{a}(L, p) a$, and then

$$
\operatorname{coth}\left(\sqrt{\eta+k_{a}^{2}(L, p)} a\right) / \operatorname{coth}\left(\sqrt{\eta+k_{b}^{2}(L, p)}(b-L)\right)>1
$$

since $\operatorname{coth}(x)$ decreases in $x$ for $x>0$. Therefore, if $k_{b}(L, p)<k_{a}(L, p)$, we have

$$
1>\frac{\sqrt{\eta+k_{b}^{2}(L, p)}}{\sqrt{\eta+k_{a}^{2}(L, p)}}=\frac{\operatorname{coth}\left(\sqrt{\eta+k_{a}^{2}(L, p)} a\right)}{\operatorname{coth}\left(\sqrt{\eta+k_{b}^{2}(L, p)}(b-L)\right)}>1,
$$

which is clearly a contradiction. We thus must have $k_{b}(L, p)>k_{a}(L, p)$ for $p$ large enough and any nonnegative $L<b-a$.

c) We only present the proof for $\rho_{b}$. Note that $k_{b}\left(L, p_{1}\right)$ satisfies

$$
\sqrt{\eta+k_{b}^{2}\left(L, p_{1}\right)} \operatorname{coth}\left(\sqrt{\eta+k_{b}^{2}\left(L, p_{1}\right)}(b-L)\right)=p_{1}
$$

and $\sqrt{\eta+k^{2}} \operatorname{coth}\left(\sqrt{\eta+k^{2}}(b-L)\right)$ increases monotonically in $k$ for $k>0$, which implies $\rho_{b}<0$ for $k \in\left(k_{\min }, k_{b}\left(L, p_{1}\right)\right)$. We then only need to prove that $\left|\rho_{b}\right|$ decreases monotonically in $k$ for $k \in\left(k_{\min }, k_{b}\left(L, p_{1}\right)\right)$. To this end, we rewrite $\rho_{b}$ as

$$
\rho_{b}=\frac{\sqrt{\eta+k^{2}} \operatorname{coth}\left(\sqrt{\eta+k^{2}}(b-L)\right)-p_{1}}{\sqrt{\eta+k^{2}} \operatorname{coth}\left(\sqrt{\eta+k^{2}} b\right)+p_{2}} \frac{\sinh \left(\sqrt{\eta+k^{2}}(b-L)\right)}{\sinh \left(\sqrt{\eta+k^{2}} b\right)} .
$$

Clearly, for $k \in\left(k_{\min }, k_{b}\left(L, p_{1}\right)\right)$ the function $\frac{p_{1}-\sqrt{\eta+k^{2}} \operatorname{coth}\left(\sqrt{\eta+k^{2}}(b-L)\right)}{p_{2}+\sqrt{\eta+k^{2}} \operatorname{coth}\left(\sqrt{\eta+k^{2}} b\right)}$ decreases monotonically in $k$. By the proof of Theorem 2.3, we also see that the function $\sinh \left(\sqrt{\eta+k^{2}}(b-L)\right) / \sinh \left(\sqrt{\eta+k^{2}} b\right)$ decreases monotonically in $k$. Therefore, as the product of these two positive decreasing functions, we obtain that $\left|\rho_{b}\right|$ decreases monotonically in $k$ for $k \in\left(k_{\min }, k_{b}\left(L, p_{1}\right)\right)$.

d) As before, we only give the proof for $\rho_{b}$. By Lemma 2.1, we know that the function $\sqrt{\eta+k^{2}} \operatorname{coth}\left(\sqrt{\eta+k^{2}}(b-L)\right)$ increases monotonically in $k$. Therefore, $\rho_{b}$ is positive if $p_{1}<\sqrt{\eta+k_{\min }^{2}} \operatorname{coth}\left(\sqrt{\eta+k_{\min }^{2}}(b-L)\right)$. We then apply a Taylor expansion of $\rho_{b}$ in $L$ for $L$ small to obtain

$$
\rho_{b}\left(k, L, \eta, p_{1}, p_{2}\right)=\rho_{b}\left(k, 0, \eta, p_{1}, p_{2}\right)-B \sqrt{\eta+k^{2}} L+O\left(L^{2}\right)
$$

with $B=\frac{\sqrt{\eta+k^{2}} \sinh \left(\sqrt{\eta+k^{2}} b\right)-p_{1} \cosh \left(\sqrt{\eta+k^{2}} b\right)}{\sqrt{\eta+k^{2}} \cosh \left(\sqrt{\eta+k^{2}} b\right)+p_{2}}$. It is easy to verify that $|B|<1$, and therefore we have

$$
\left|\rho_{b}\left(k, L, \eta, p_{1}, p_{2}\right)-\rho_{b}\left(k, 0, \eta, p_{1}, p_{2}\right)\right|<\sqrt{\eta+k^{2}} L+O\left(L^{2}\right) .
$$

Since $\rho_{b}\left(k, 0, \eta, p_{1}, p_{2}\right)$ clearly increases monotonically in $k$, we obtain that $\rho_{b}$ is asymptotically increasing in $k$ for $k_{\min }<k<c L^{-\frac{1}{2}}$.

THEOREM 3.3 (T0 asymptotics). With overlap $L>0$, the parallel Schwarz method (1.2) with Taylor transmission condition of order 0 given in (3.4) satisfies, asymptotically for $L \rightarrow 0$, the convergence factor estimate

$$
\max _{k \in \mathbb{K}}\left|\rho_{T 0}\left(k, L, \eta, p_{1}^{T 0}, p_{2}^{T 0}, a, b\right)\right|=1-8 G_{0}^{\frac{1}{2}} L^{\frac{1}{2}}+O(L),
$$




\section{ETNA}

Kent State University and

Johann Radon Institute (RICAM)

where $G_{0}=G(0, \eta, a, b)$. If there is no overlap, then the asymptotic convergence factor estimate for $k_{\max } \rightarrow \infty$ is

$$
\max _{k \in \mathbb{K}}\left|\rho_{T 0}\left(k, 0, \eta, p_{1}^{T 0}, p_{2}^{T 0}, a, b\right)\right|=1-8 G_{0} k_{\max }^{-1}+O\left(k_{\max }^{-2}\right) .
$$

Proof. A direct calculation shows that $\rho_{a p p}\left(k, L, \eta, p_{1}^{T 0}, p_{2}^{T 0}\right)$ attains its unique interior maximum asymptotically at $k_{T 0}=2 G_{0}^{\frac{1}{2}} L^{-\frac{1}{2}}$. We then rewrite $\rho_{T 0}$ as

$$
\rho_{T 0}=\rho_{b}\left(k, L, \eta, p_{1}^{T 0}, p_{2}^{T 0}\right) \rho_{a}\left(k, L, \eta, p_{1}^{T 0}, p_{2}^{T 0}\right) .
$$

Applying result d) of Lemma 3.2 implies that the convergence factor $\rho_{T 0}$, as the product of $\rho_{a}$ and $\rho_{b}$, is increasing in $k$ asymptotically for $k_{\min }<k<k_{T 0}$. When $k>k_{T 0}$, by Theorem 3.1 we conclude that the convergence factor $\rho_{T 0}$ is asymptotically decreasing in $k$ since the approximate convergence factor $\rho_{a p p}$ decreases. Thus, the convergence factor $\rho_{T 0}$ attains its maximum asymptotically at $k_{T 0}$. A Taylor expansion of the convergence factor $\rho_{T 0}$ at $k_{T 0}$ gives then the first result. For the second one, from the proof of Lemma 3.2 we know that $\rho_{a}\left(k, 0, \eta, p_{1}^{T 0}, p_{2}^{T 0}\right)$ and $\rho_{b}\left(k, 0, \eta, p_{1}^{T 0}, p_{2}^{T 0}\right)$ increase monotonically in $k$ for $k>0$. Therefore, the convergence factor $\rho_{T 0}\left(k, 0, \eta, p_{1}^{T 0}, p_{2}^{T 0}\right)$, as the product of these two, increases monotonically in $k$ for $k>0$, and thus the second result follows from the Taylor expansion of $\rho_{T 0}$ at $k_{\max }$.

Similarly, Taylor transmission conditions of order 2 (T2) are obtained by truncating the Taylor expansion of $\sigma_{i}(k)$ at $k=0$ at second order,

$$
\begin{array}{ll}
p_{1}^{T 2}=p_{1}^{T 0}, & q_{1}=\frac{1}{2 \sqrt{\eta}} \frac{1-4(b-L) \sqrt{\eta} e^{-2 \sqrt{\eta}(b-L)}-e^{-4 \sqrt{\eta}(b-L)}}{\left(1-e^{-2 \sqrt{\eta}(b-L)}\right)^{2}}, \\
p_{2}^{T 2}=p_{2}^{T 0}, & q_{2}=\frac{1}{2 \sqrt{\eta}} \frac{1-4 a \sqrt{\eta} e^{-2 \sqrt{\eta} a}-e^{-4 \sqrt{\eta} a}}{\left(1-e^{-2 \sqrt{\eta} a}\right)^{2}} .
\end{array}
$$

However, the calculations involved in the theoretical analysis of the resulting Schwarz methods becomes very complicated, and we will see that a better performance can be obtained through optimization.

3.2. Optimized transmission condition of order 0 . In this section we impose constraints on $p_{i}, q_{i}$ as follows: $p_{i}=p$ with $p$ a positive constant and $q_{i}=0$. In this case the convergence factor of the parallel Schwarz algorithm (1.2) is

$$
\begin{aligned}
\rho_{O O 0}(k, L, \eta, p, a, b)= & \frac{\sqrt{\eta+k^{2}} \cosh \left(\sqrt{\eta+k^{2}}(b-L)\right)-p \sinh \left(\sqrt{\eta+k^{2}}(b-L)\right)}{\sqrt{\eta+k^{2}} \cosh \left(\sqrt{\eta+k^{2}}(a+L)\right)+p \sinh \left(\sqrt{\eta+k^{2}}(a+L)\right)} \times \\
& \frac{\sqrt{\eta+k^{2}} \cosh \left(\sqrt{\eta+k^{2}} a\right)-p \sinh \left(\sqrt{\eta+k^{2}} a\right)}{\sqrt{\eta+k^{2}} \cosh \left(\sqrt{\eta+k^{2}} b\right)+p \sinh \left(\sqrt{\eta+k^{2}} b\right)},
\end{aligned}
$$

and we look for the best transmission parameters by studying the min-max problem

$$
\min _{p>0}\left(\max _{k \in \mathbb{K}}\left|\rho_{O O 0}(k, L, \eta, p, a, b)\right|\right),
$$

where $\mathrm{OO} 0$ stands for "Optimized of Order 0"; see for example [14]. However, unlike in the infinite domain decomposition analysis [14], the above min-max problem can not be solved directly. Here, we use the technique applied in [20,23], that is to say, when the frequency $k$ is small, we use the exact convergence factor $\rho_{O O 0}$ directly; when the frequency $k$ is large, we 
use the approximate convergence factor $\rho_{a p p}$ instead of the exact convergence factor. We can prove that we asymptotically solve the min-max problem (3.5):

THEOREM 3.4 (OO0, overlapping case). For $L>0$, the solution $p^{*}$ to

$$
\rho_{O O 0}\left(k_{\min }, L, \eta, p^{*}, a, b\right)=\rho_{a p p}\left(\bar{k}^{*}, L, \eta, p^{*}, p^{*}\right)
$$

is given asymptotically by

$$
p^{*}=2^{\frac{1}{3}} G_{\min }^{\frac{2}{3}} L^{-\frac{1}{3}},
$$

where $\bar{k}^{*}$ is the location of the unique interior maximum of $\rho_{\text {app }}$ and asymptotically given by $\bar{k}^{*}=2^{\frac{2}{3}} G_{\min }^{\frac{1}{3}} L^{-\frac{2}{3}}$. The above $p^{*}$ solves asymptotically the min-max problem (3.5) and the corresponding convergence factor satisfies the following estimate as $L$ goes to zero:

$$
\max _{k \in \mathbb{K}}\left|\rho_{O O 0}\left(k, L, \eta, p^{*}, a, b\right)\right|=1-2^{\frac{8}{3}} G_{\min }^{\frac{1}{3}} L^{\frac{1}{3}}+O\left(L^{\frac{2}{3}}\right) .
$$

Proof. A direct calculation shows that the unique interior maximum point of $\rho_{a p p}$ with respect to $k$ is given by $\bar{k}(L, \eta, p)=\sqrt{L\left(2 p+L\left(p^{2}-\eta\right)\right)} / L$. We then need to find the asymptotic expansion of $p^{*}$ for $L$ small from (3.6). We make the ansatz $p=C_{p} L^{-\alpha}$ for $\alpha>0$ and define $p^{*}=C_{p}^{*} L^{-\alpha^{*}}$. We first consider the case $\alpha<1$. In this case, we have $\bar{k}(L, \eta, p) \sim \sqrt{2 C_{p}} L^{-\frac{1+\alpha}{2}}$ for $L$ small. Expanding the left-hand side of (3.6) in $L$ for $L$ small yields

$$
\rho_{O O 0}\left(k_{\min }, L, \eta, p^{*}, a, b\right)=1-\frac{8}{C_{p}^{*}} G_{\min } L^{\alpha^{*}}+o\left(L^{\alpha^{*}}\right) .
$$

Expanding the right-hand side of (3.6) in $L$ for $L$ small yields

$$
\rho_{\text {app }}\left(\bar{k}^{*}, L, \eta, p^{*}, p^{*}\right)=1-4 \sqrt{2 C_{p}^{*}} L^{\frac{1-\alpha^{*}}{2}}+o\left(L^{\frac{1-\alpha^{*}}{2}}\right) .
$$

Since (3.6) holds for all $L>0$, we must have an equality of the leading order terms, i.e., $\frac{8}{C_{p}^{*}} G_{\min } L^{\alpha^{*}}=4 \sqrt{2 C_{p}^{*}} L^{\frac{1-\alpha^{*}}{2}}$, which leads to the solution $\alpha^{*}=\frac{1}{3}, C_{p}^{*}=2^{\frac{1}{3}} G_{\min }^{\frac{2}{3}}$ and thus to the parameter $p^{*}$ in (3.7). With this asymptotic expression of $p^{*}$, the interior maximum $\bar{k}^{*}$ behaves asymptotically like

$$
\bar{k}^{*}=2^{\frac{2}{3}} G_{\min }^{\frac{1}{3}} L^{-\frac{2}{3}} .
$$

Similarly, we can show that if $\alpha \geq 1$, then there is no valid solution $\alpha^{*}$.

We then show the second result. In this case, $k_{a}\left(L, p^{*}\right)$ behaves asymptotically as $k_{a}\left(L, p^{*}\right)=C_{p}^{*} L^{-\frac{1}{3}}$ for $L$ small enough. By result c) of Lemma 3.2, we know that the convergence factor $\rho_{O O 0}\left(k, L, \eta, p^{*}, a, b\right)$ decreases monotonically in $k$ for $k \in\left(k_{\min }, k_{a}\left(L, p^{*}\right)\right)$. This implies that $k_{\min }$ is a possible maximum point for $L$ small.

If $k>k_{a}\left(L, p^{*}\right)$, then we know that

$$
\begin{aligned}
& \left|\rho_{O O 0}\left(k, L, \eta, p^{*}, a, b\right)-\rho_{a p p}\left(k, L, \eta, p^{*}, p^{*}\right)\right| \\
& \quad \leq 2\left(e^{-2 \sqrt{\eta+k_{a}^{2}\left(L, p^{*}\right) b}}+e^{-2 \sqrt{\eta+k_{a}^{2}\left(L, p^{*}\right)}(a+L)}\right) .
\end{aligned}
$$

Hence $\rho_{O O 0}\left(k, L, \eta, p^{*}, a, b\right)$ attains its unique interior maximum asymptotically at the point $\bar{k}^{*}=2^{\frac{2}{3}} G_{\min }^{\frac{1}{3}} L^{-\frac{2}{3}}$. 
We can now show that $p^{*}$ given by (3.7) solves the min-max problem (3.5) asymptotically. Assuming $p=C_{p} L^{-\alpha}$, we only need to show that if $\alpha \neq \alpha^{*}=\frac{1}{3}$, or $C_{p} \neq C_{p}^{*}$, we have $\max _{k}\left|\rho_{O O 0}(k, L, \eta, p, a, b)\right|>\max _{k}\left|\rho_{O O 0}\left(k, L, \eta, p^{*}, a, b\right)\right|$ for $L$ small enough. We show that when $\alpha \neq \alpha^{*}$, the asymptotic order in the expansion of the convergence factor is amplified. When $\alpha<\alpha^{*}$, we have

$$
\rho_{a p p}(\bar{k}(L, \eta, p), L, \eta, p, p)=1-4 \sqrt{2 C_{p}} L^{\frac{1-\alpha}{2}}+o\left(L^{\frac{1-\alpha}{2}}\right),
$$

and by Theorem 3.1, $\rho_{O O 0}(\bar{k}(L, \eta, p), L, \eta, p, a, b)$ behaves the same way. Thus we have for $L$ small enough that

$$
\rho_{O O 0}(\bar{k}(L, \eta, p), L, \eta, a, b)>\rho_{O O 0}\left(\bar{k}^{*}, L, \eta, p^{*}, a, b\right) .
$$

When $\alpha>\alpha^{*}$, we consider the convergence factor at $k_{\min }$ and obtain

$$
\rho_{O O 0}\left(k_{\min }, L, \eta, p, a, b\right)=1-\frac{\sqrt{8}}{C_{p}} G_{\min } L^{\alpha}+o\left(L^{\alpha}\right),
$$

which is greater than $\rho_{O O 0}\left(k_{\min }, L, \eta, p^{*}, a, b\right)$ for $L$ small enough.

We finally consider the case $\alpha=\alpha^{*}$ but $C_{p} \neq C_{p}^{*}$. By the asymptotic expansions above we find that $\rho_{O O 0}\left(k_{\min }, L, \eta, p, a, b\right)>\rho_{O O 0}\left(k_{\min }, L, \eta, p^{*}, a, b\right)$ if $C_{p}>C_{p}^{*}$ and $\rho_{O O 0}(\bar{k}(L, \eta, p), L, \eta, p, a, b)>\rho_{O O 0}\left(\bar{k}\left(L, \eta, p^{*}\right), L, \eta, p^{*}, a, b,\right)$ if $C_{p}<C_{p}^{*}$, which concludes the proof of the asymptotic optimality. To show the convergence factor estimate, we only insert $C_{p}^{*}$ and $\alpha^{*}$ into (3.8).

THEOREM 3.5 (OO0, nonoverlapping case). When the overlap $L=0$, the solution $\bar{p}$ to

$$
\rho_{O O 0}\left(k_{\min }, 0, \eta, \bar{p}, a, b\right)=\rho_{O O 0}\left(k_{\max }, 0, \eta, \bar{p}, a, b\right)
$$

is given in closed form by

$$
\bar{p}=\left(\frac{f_{\min }^{a} f_{\min }^{b} f_{\max }^{a} f_{\max }^{b}}{-f_{\min }^{a}-f_{\min }^{b}+f_{\max }^{a}+f_{\max }^{b}}\left(-\frac{1}{f_{\max }^{a}}-\frac{1}{f_{\max }^{b}}+\frac{1}{f_{\min }^{a}}+\frac{1}{f_{\min }^{b}}\right)\right)^{\frac{1}{2}}
$$

with

$$
\begin{aligned}
& f_{\min }^{a}=f\left(a \sqrt{\eta+k_{\min }^{2}}\right) / a, \quad \quad f_{\min }^{b}=f\left(b \sqrt{\eta+k_{\min }^{2}}\right) / b, \\
& f_{\max }^{a}=f\left(a \sqrt{\eta+k_{\max }^{2}}\right) / a, \quad \text { and } \quad f_{\max }^{b}=f\left(b \sqrt{\eta+k_{\max }^{2}}\right) / b \text {, }
\end{aligned}
$$

where $f(x)$ is defined in Lemma 2.1. In addition, $\bar{p}$ is, for $k_{\max } \rightarrow+\infty$, asymptotically given by

$$
\bar{p}=\sqrt{2 G_{\min }} k_{\max }^{\frac{1}{2}}
$$

The above $\bar{p}$ solves asymptotically the min-max problem (3.5), and we get for $k_{\max } \rightarrow \infty$ the convergence factor estimate

$$
\max _{k \in \mathbb{K}}\left|\rho_{O O 0}(k, 0, \eta, \bar{p}, a, b)\right|=1-2^{\frac{5}{2}} G_{\min }^{\frac{1}{2}} k_{\max }^{-\frac{1}{2}}+O\left(k_{\max }^{-1}\right) .
$$

Proof. We rewrite the convergence factor $\rho_{O O 0}(k, 0, \eta, p, a, b)$ as

$$
\rho_{O O 0}(k, 0, \eta, p, a, b)=\rho_{b}(k, 0, \eta, p, p) \rho_{a}(k, 0, \eta, p, p)
$$


Solving directly the equi-oscillation equation (3.9) gives the closed form of the parameter $\bar{p}$. Note that $\operatorname{coth}(x) \rightarrow 1$ converges faster than any polynomial as $x \rightarrow \infty$, and an asymptotic expansion in $k_{\max }$ of $\bar{p}$ gives $\bar{p}=\sqrt{2 G_{\min }} k_{\max }^{\frac{1}{2}}$ for $k_{\max } \rightarrow \infty$.

From the result c) and the proof of Lemma 3.2, we know that $\rho_{O O 0}(k, 0, \eta, \bar{p}, a, b)$ is monotonically decreasing in $k$ for $k \in\left(k_{\min }, k_{a}(0, \bar{p})\right)$ and is monotonically increasing in $k$ for $k \in\left(k_{b}(0, \bar{p}), k_{\max }\right)$, and $\rho_{O O 0}(k, 0, \eta, \bar{p}, a, b)$ is positive in these two intervals. Thus, in between $k_{a}(0, \bar{p})$ and $k_{b}(0, \bar{p})$, the convergence factor $\rho_{O O 0}$ attains its minimum. We show next that when $k$ lies in between $k_{a}(0, \bar{p})$ and $k_{b}(0, \bar{p})$, then

$$
\left|\rho_{O O 0}(k, 0, \eta, \bar{p}, a, b)\right|<\rho_{O O 0}\left(k_{\min }, 0, \eta, \bar{p}, a, b\right) \quad \text { for } k_{\max } \text { large enough. }
$$

Note that both $k_{a}(0, \bar{p})$ and $k_{b}(0, \bar{p})$ behave asymptotically as

$$
k_{a}(0, \bar{p}) \approx k_{b}(0, \bar{p}) \sim \sqrt{2 G_{\min }} k_{\max }^{\frac{1}{2}} .
$$

When $k$ lies in between $k_{a}(0, \bar{p})$ and $k_{b}(0, \bar{p})$, we have from Theorem 3.1 that

$$
\left|\rho_{O O 0}(k, 0, \eta, \bar{p}, a, b)-\rho_{a p p}(k, 0, \eta, \bar{p}, \bar{p})\right| \leq 2\left(e^{-2 \sqrt{\eta+k_{a}^{2}(0, \bar{p})} b}+e^{-2 \sqrt{\eta+k_{a}^{2}(0, \bar{p})} a}\right) .
$$

Incorporating the above estimate into the fact that $\rho_{a p p}(k, \eta, 0, \bar{p}, \bar{p})$ attains its unique minimum asymptotically at $\sqrt{2 G_{\min }} k_{\max }^{\frac{1}{2}}$ with a minimum value 0 , we obtain, as $k_{\max } \rightarrow \infty$, that $\left|\rho_{O O 0}(k, 0, \eta, \bar{p}, a, b)\right|<\rho_{O O 0}\left(k_{\min }, 0, \eta, \bar{p}, a, b\right)$ for $k \in\left(k_{a}(0, \bar{p}), k_{b}(0, \bar{p})\right)$.

Next, we only need to show that when $p \neq \bar{p}$, there exists a $k^{*}$ such that

$$
\rho_{O O 0}\left(k^{*}, 0, \eta, p, a, b\right)>\rho_{O O 0}\left(k_{\min }, 0, \eta, \bar{p}, a, b\right) .
$$

Let $p:=C_{p} k_{\max }^{\alpha}$ and $\bar{p}:=\bar{C}_{p} k_{\max }^{\bar{\alpha}}$ with $\bar{C}_{p}=\sqrt{2 G_{\min }}$ and $\bar{\alpha}=\frac{1}{2}$. We show first that if $\alpha \neq \bar{\alpha}$, then the asymptotic order in the expansion of the convergence factor is amplified. If $\alpha>\bar{\alpha}$, then we take $k^{*}=k_{\min }$. We have

$$
\rho_{O O 0}\left(k_{\min }, 0, \eta, p, a, b\right)=1-8 \frac{G_{\min }}{C_{p}} k_{\max }^{-\alpha}+o\left(k_{\max }^{-\alpha}\right) .
$$

If $\alpha<\bar{\alpha}$, then we take $k^{*}=k_{\max }$. We have by Theorem 3.1,

$$
\rho_{O O 0}\left(k_{\max }, 0, \eta, p, a, b\right)=1-4 C_{p} k_{\max }^{\alpha-1}+o\left(k_{\max }^{\alpha-1}\right) .
$$

Therefore, in each case above we get $\max _{k}\left|\rho_{O O 0}(k, 0, \eta, p, a, b)\right|>1-c k_{\max }^{-\delta}$ with $\delta>\frac{1}{2}$. We then consider the case $\alpha=\bar{\alpha}$ but $C_{p} \neq \bar{C}_{p}$. By (3.11) we see that

$$
\left|\rho_{O O 0}\left(k_{\min }, 0, \eta, p, a, b\right)\right|>\left|\rho_{O O 0}\left(k_{\min }, 0, \eta, \bar{p}, a, b\right)\right| \quad \text { if } C_{p}>\bar{C}_{p} .
$$

By (3.12) we see that

$$
\left|\rho_{O O 0}\left(k_{\max }, 0, \eta, p, a, b\right)\right|>\left|\rho_{O O 0}\left(k_{\max }, 0, \eta, \bar{p}, a, b\right)\right| \quad \text { if } C_{p}<\bar{C}_{p} .
$$

Hence the maximum of the convergence factor $\left|\rho_{O O 0}(k, 0, \eta, p, a, b)\right|$ attains its minimum at $p=\bar{p}$ asymptotically. Inserting the asymptotic formula of $\bar{p}$ into $\rho_{O O 0}\left(k_{\min }, 0, \eta, \bar{p}, a, b\right)$ and asymptotically expanding it with respect to $k_{\max }$ for $k_{\max }$ large enough gives (3.10). 
3.3. Optimized transmission condition of order 2. In this section we impose on $p_{i}$ and $q_{i}$ the constraints

$$
p_{i}=p, \quad q_{i}=q, \quad i=1,2,
$$

with $p$ and $q$ positive parameters. To determine the best parameters $p$ and $q$, we thus need to solve the min-max problem

$$
\min _{p>0, q>0}\left(\max _{k \in \mathbb{K}}\left|\rho_{O O 2}(k, L, \eta, p, q, a, b)\right|\right),
$$

with

$$
\begin{aligned}
\rho_{O O 2}( & k, L, \eta, p, q, a, b) \\
= & \frac{\sqrt{\eta+k^{2}} \cosh \left(\sqrt{\eta+k^{2}}(b-L)\right)-\left(p+q k^{2}\right) \sinh \left(\sqrt{\eta+k^{2}}(b-L)\right)}{\sqrt{\eta+k^{2}} \cosh \left(\sqrt{\eta+k^{2}}(a+L)\right)+\left(p+q k^{2}\right) \sinh \left(\sqrt{\eta+k^{2}}(a+L)\right)} \times \\
& \frac{\sqrt{\eta+k^{2}} \cosh \left(\sqrt{\eta+k^{2}} a\right)-\left(p+q k^{2}\right) \sinh \left(\sqrt{\eta+k^{2}} a\right)}{\sqrt{\eta+k^{2}} \cosh \left(\sqrt{\eta+k^{2}} b\right)+\left(p+q k^{2}\right) \sinh \left(\sqrt{\eta+k^{2}} b\right)},
\end{aligned}
$$

where $\mathrm{OO} 2$ stands for "Optimized of Order 2", a second-order transmission condition that is also known as the optimized Ventcell transmission condition in the literature. Again, we need the technique used in $[20,23]$ for the analysis to obtain the following results.

THEOREM 3.6 (OO2, overlapping case). For $L>0$, the solutions $p^{*}$ and $q^{*}$ to

$$
\begin{aligned}
\rho_{O O 2}\left(k_{\min }, L, \eta, p^{*}, q^{*}, a, b\right) & =\rho_{a p p}\left(\bar{k}_{1}^{*}, L, \eta, p^{*}+q^{*}\left(\bar{k}_{1}^{*}\right)^{2}, p^{*}+q^{*}\left(\bar{k}_{1}^{*}\right)^{2}\right) \\
& =\rho_{a p p}\left(\bar{k}_{2}^{*}, L, \eta, p^{*}+q^{*}\left(\bar{k}_{2}^{*}\right)^{2}, p^{*}+q^{*}\left(\bar{k}_{2}^{*}\right)^{2}\right)
\end{aligned}
$$

are asymptotically given by

$$
p^{*}=2^{\frac{1}{5}} G_{\min }^{\frac{4}{5}} L^{-\frac{1}{5}}, \quad q^{*}=2^{-\frac{3}{5}} G_{\min }^{-\frac{2}{5}} L^{\frac{3}{5}},
$$

where $\bar{k}_{1}^{*}$ and $\bar{k}_{2}^{*}$ are the locations of the interior maxima of $\rho_{\text {app }}$ which can be expressed asymptotically as $\bar{k}_{1}^{*}=2^{\frac{2}{5}} G_{\min }^{\frac{3}{5}} L^{-\frac{2}{5}}$ and $\bar{k}_{2}^{*}=2^{\frac{4}{5}} G_{\min }^{\frac{1}{5}} L^{-\frac{4}{5}}$. The above $p^{*}$ and $q^{*}$ solve asymptotically the min-max problem (3.13), and the corresponding convergence factor satisfies the estimate as $L \rightarrow 0$

$$
\max _{k \in \mathbb{K}}\left|\rho_{O O 2}\left(k, L, \eta, p^{*}, q^{*}, a, b\right)\right|=1-2^{\frac{14}{5}} G_{\min }^{\frac{1}{5}} L^{\frac{1}{5}}+O\left(L^{\frac{2}{5}}\right) .
$$

Proof. From the derivative of $\rho_{a p p}\left(k, L, \eta, p+q k^{2}, p+q k^{2}\right)$ in $k$, we get

$$
\begin{aligned}
\bar{k}_{1,2}(L, \eta, p, q) & =\frac{1}{q} \sqrt{\frac{L+2 q-2 L p q \mp \sqrt{\Phi}}{2 L}}, \quad \text { with } \\
\Phi & =L^{2}+4 L q-4 L^{2} p q+4 q^{2}-16 L p q^{2}+16 L q^{3} \eta+4 L^{2} q^{2} \eta .
\end{aligned}
$$

We make the ansatz $p:=C_{p} L^{-\frac{1}{5}}$ and $q:=C_{q} L^{\frac{3}{5}}$. Inserting this ansatz into $\bar{k}_{1,2}$ and expanding for $L$ small, we obtain $\bar{k}_{1}(L, \eta, p, q) \sim \sqrt{\frac{C_{p}}{C_{q}}} L^{-\frac{2}{5}}$ and $\bar{k}_{2}(L, \eta, p, q) \sim \sqrt{\frac{2}{C_{q}}} L^{-\frac{4}{5}}$. Again, using the same ansatz in the following expansions for $L$ small, we obtain

$$
\begin{array}{r}
\rho_{O O 2}\left(k_{\min }, L, \eta, p, q, a, b\right)=1-\frac{8 G_{\min }}{C_{p}} L^{\frac{1}{5}}+O\left(L^{-\frac{2}{5}}\right), \\
\rho_{a p p}\left(\bar{k}_{1}, L, \eta, p+q \bar{k}_{1}^{2}, p+q \bar{k}_{1}^{2}\right)=1-8 \sqrt{C_{p} C_{q}} L^{\frac{1}{5}}+O\left(L^{\frac{2}{5}}\right),
\end{array}
$$


and

$$
\rho_{a p p}\left(\bar{k}_{2}, L, \eta, p+q \bar{k}_{2}^{2}, p+q \bar{k}_{2}^{2}\right)=1-\frac{4 \sqrt{2}}{\sqrt{C_{q}}} L^{\frac{1}{5}}+O\left(L^{\frac{2}{5}}\right) .
$$

By Theorem 3.1, we conclude that $\rho_{\mathrm{OO} 2}(k, L, \eta, p, q, a, b)$ behaves asymptotically the same way as $\rho_{a p p}\left(k, L, \eta, p+q k^{2}, p+q k^{2}\right)$ at $\bar{k}_{1,2}$. Since (3.14) holds for any small $L>0$, setting the coefficients of the term $L^{\frac{1}{5}}$ in the three expansions above to be equal to each other, we obtain

$$
-\frac{8 G_{\min }}{C_{p}}=-8 \sqrt{C_{p} C_{q}}=-4 \sqrt{\frac{2}{C_{q}}},
$$

whose solution $C_{p}^{*}, C_{q}^{*}$ leads to the announced results for $p^{*}, q^{*}$, and $\bar{k}_{1,2}^{*}$.

Inserting the asymptotic values of $p^{*}, q^{*}$, and $k=k_{\min }$ into the convergence factor $\rho_{O O 2}(k, L, \eta, p, q)$ and expanding for $L$ small, we obtain

$$
\rho_{O O 2}\left(k_{\min }, L, \eta, p^{*}, q^{*}, a, b\right)=1-2^{\frac{14}{5}} G_{\min }^{\frac{1}{5}} L^{\frac{1}{5}}+O\left(L^{\frac{2}{5}}\right) .
$$

Thus, we only need to show that $p^{*}, q^{*}$ solve the min-max problem (3.13) asymptotically.

Note that

$$
\rho_{O O 2}(k, L, \eta, p, q, a, b)=\rho_{b}\left(k, L, \eta, p+q k^{2}, p+q k^{2}\right) \rho_{a}\left(k, L, \eta, p+q k^{2}, p+q k^{2}\right) .
$$

We denote by $k_{b}\left(L, p^{*}, q^{*}\right)$ the smaller positive zero of $\rho_{b}\left(k, L, \eta, p^{*}+q^{*} k^{2}, p^{*}+q^{*} k^{2}\right)$ and by $k_{a}\left(L, p^{*}, q^{*}\right)$ the smaller positive zero of $\rho_{a}\left(k, L, \eta, p^{*}+q^{*} k^{2}, p^{*}+q^{*} k^{2}\right)$. Then similar to the proof of Lemma 3.2, we obtain $k_{a}\left(L, p^{*}, q^{*}\right)<k_{b}\left(L, p^{*}, q^{*}\right)$, and both of them behave as $p^{*}$ for $L$ small enough. By a similar argument as for the result c) of Lemma 3.2, we can show that $\rho_{b, a}\left(k, L, \eta, p^{*}+q^{*} k^{2}, p^{*}+q^{*} k^{2}\right)$ are negative and increasing in $k$ for $k \in\left(k_{\min }, k_{a}\left(L, p^{*}, q^{*}\right)\right)$. Thus, $\rho_{O O 2}\left(k, L, \eta, p^{*}, q^{*}, a, b\right)$ is positive and asymptotically decreasing in $k$ for $k \in\left(k_{\min }, k_{a}\left(L, p^{*}, q^{*}\right)\right)$, and $k_{\min }$ is a possible maximum point for $L$ small enough. For $k \in\left(k_{a}\left(L, p^{*}, q^{*}\right), k_{\max }\right)$, noting that $\rho_{a p p}\left(k, L, \eta, p^{*}+q^{*} k^{2}, p^{*}+q^{*} k^{2}\right)$ has two interior maximum points $\bar{k}_{1}^{*}$ and $\bar{k}_{2}^{*}$, by Theorem 3.1 , we know that $\rho_{O O 2}\left(k, L, \eta, p^{*}, q^{*}, a, b\right)$ attains its interior maxima at $\bar{k}_{1,2}^{*}$ asymptotically.

We then only need to show that if $(p, q) \neq\left(p^{*}, q^{*}\right)$, then there exists a $k^{*}$ such that for $L>0$ small enough

$$
\rho_{O O 2}\left(k^{*}, L, \eta, p, q, a, b\right)>1-\frac{8 G_{\min }}{C_{p}^{*}} L^{\frac{1}{5}} .
$$

To this end, let $p:=C_{p} L^{-\alpha}$ and $q:=C_{q} L^{\beta}$. We consider first the case $(\alpha, \beta) \neq\left(\alpha^{*}, \beta^{*}\right)$ $=\left(\frac{1}{5}, \frac{3}{5}\right)$. It is sufficient to treat the following cases:

a) $\alpha>\alpha^{*}, \beta>-\alpha$. In this case, at $k^{*}=k_{\min }$, we have

$$
\rho_{O O 2}\left(k^{*}, L, \eta, p, q, a, b\right)=1-\frac{8 G_{\min }}{C_{p}} L^{\alpha}-4 G_{\min } L+o\left(L^{\min \{\alpha, 1\}}\right) .
$$

b) $\alpha>\alpha^{*}, \beta=-\alpha$. In this case, at $k^{*}=k_{\min }$, we have

$$
\rho_{O O 2}\left(k^{*}, L, \eta, p, q, a, b\right)=1-\frac{8 G_{\min }}{C_{p}+C_{q} k_{\min }^{2}} L^{\alpha}-4 G_{\min } L+o\left(L^{\min \{\alpha, 1\}}\right) .
$$


c) $\alpha>\alpha^{*}, \beta<-\alpha$. In this case, at $k^{*}=k_{\min }$, we have

$$
\rho_{O O 2}\left(k^{*}, L, \eta, p, q, a, b\right)=1-\frac{8 G_{\min }}{C_{q} k_{\min }^{2}} L^{-\beta}-4 G_{\min } L+o\left(L^{\min \{-\beta, 1\}}\right) .
$$

d) $\alpha=\alpha^{*}, \beta^{*}<\beta \leq 1$. In this case, at $k^{*}=C_{k} L^{-\frac{\alpha+\beta}{2}}$, we have

$$
\begin{aligned}
\rho_{\text {app }}\left(k^{*}, L, \eta, p+q\left(k^{*}\right)^{2}, p+q\left(k^{*}\right)^{2}\right) \\
\quad=1-\frac{4\left(C_{p}+C_{q} C_{k}^{2}\right)}{C_{k}} L^{\frac{\beta-\alpha}{2}}-2 C_{k} L^{-\frac{2-\alpha-\beta}{2}}+o\left(L^{\frac{\beta-\alpha}{2}}\right) .
\end{aligned}
$$

e) $\alpha=\alpha^{*}, \beta>1$. In this case, at $k^{*}=C_{k} L^{-\frac{3}{5}}$, we have

$$
\rho_{\text {app }}\left(k^{*}, L, \eta, p+q\left(k^{*}\right)^{2}, p+q\left(k^{*}\right)^{2}\right)=1-2\left(\frac{2 C_{p}}{C_{k}}+C_{k}\right) L^{\frac{2}{5}}+o\left(L^{\frac{2}{5}}\right) .
$$

f) $\alpha \leq \alpha^{*}, \beta<\beta^{*}$. In this case, we consider $k^{*}=C_{k} L^{-\frac{\beta+1}{2}}$. We obtain

$$
\rho_{\text {app }}\left(k^{*}, L, \eta, p+q\left(k^{*}\right)^{2}, p+q\left(k^{*}\right)^{2}\right)=1-2\left(\frac{2}{C_{q} C_{k}}+C_{k}\right) L^{\frac{1-\beta}{2}}+o\left(L^{\frac{1-\beta}{2}}\right) .
$$

g) $\alpha<\alpha^{*}, \beta>\beta^{*}$. In this case, at $k^{*}=C_{k} L^{-\frac{2}{5}}$, we have

$$
\begin{aligned}
\rho_{a p p} & \left(k^{*}, L, \eta, p+q\left(k^{*}\right)^{2}, p+q\left(k^{*}\right)^{2}\right) \\
& =1-4\left(\frac{C_{p}}{C_{k}} L^{\frac{2}{5}-\alpha}+C_{q} C_{k} L^{\beta-\frac{2}{5}}\right)-2 C_{k} L^{\frac{3}{5}}+o\left(L^{\min \left\{\frac{2}{5}-\alpha, \beta-\frac{2}{5}, \frac{3}{5}\right\}}\right) .
\end{aligned}
$$

h) $\alpha<\alpha^{*}, \beta=\beta^{*}$. In this case, at $k^{*}=C_{k} L^{-\frac{\beta+\alpha}{2}}$, we have

$$
\rho_{a p p}\left(k^{*}, L, \eta, p+q\left(k^{*}\right)^{2}, p+q\left(k^{*}\right)^{2}\right)=1-4\left(\frac{C_{p}}{C_{k}}+C_{q} C_{k}\right) L^{\frac{\beta-\alpha}{2}}+o\left(L^{\frac{\beta-\alpha}{2}}\right) .
$$

Note that in the cases d)-h) we get the expansions of $\rho_{a p p}$ at $k^{*}$. By Theorem 3.1 we know that in each case $\rho_{\mathrm{OO} 2}\left(k^{*}, L, \eta, p, q, a, b\right)$ has the same asymptotic expansion as the function $\rho_{\text {app }}\left(k^{*}, L, \eta, p+q\left(k^{*}\right)^{2}, p+q\left(k^{*}\right)^{2}\right)$. We therefore see that in each case above, at the given frequency $k^{*}$, the convergence factor $\rho_{O O 2}\left(k^{*}, L, \eta, p, q, a, b\right)$ behaves asymptotically like $1-C L^{\delta}$ with $\delta>\frac{1}{5}$.

Thus, it remains to consider the case $(\alpha, \beta)=\left(\alpha^{*}, \beta^{*}\right)$ but $\left(C_{p}, C_{q}\right) \neq\left(C_{p}^{*}, C_{q}^{*}\right)$. From (3.15) we see that $\rho_{\mathrm{OO} 2}\left(k_{\min }, L, \eta, p, q, a, b\right)>\rho_{\mathrm{OO} 2}\left(k_{\min }, L, \eta, p^{*}, q^{*}, a, b\right)$ for $L>0$ small enough if $C_{p}>C_{p}^{*}$. From (3.17) together with Theorem 3.1, we see that

$$
\rho_{\mathrm{OO} 2}\left(\bar{k}_{2}, L, \eta, p, q, a, b\right)>\rho_{\mathrm{OO} 2}\left(\bar{k}_{2}^{*}, L, \eta, p^{*}, q^{*}, a, b\right)
$$

for $L>0$ small enough if $C_{q}>C_{q}^{*}$. From (3.16) together with Theorem 3.1, we conclude that $\rho_{\mathrm{OO} 2}\left(\bar{k}_{1}, L, \eta, p, q, a, b\right)>\rho_{\mathrm{OO} 2}\left(\bar{k}_{1}^{*}, L, \eta, p^{*}, q^{*}, a, b\right)$ for $L>0$ small enough if $C_{p}<C_{p}^{*}$ or $C_{q}<C_{q}^{*}$, which ends the proof.

THEOREM 3.7 (OO2, nonoverlapping case). For vanishing overlap, $L=0$, the solutions $\bar{p}$ and $\bar{q}$ to

$$
\begin{aligned}
\rho_{O O 2}\left(k_{\min }, 0, \eta, \bar{p}, \bar{q}, a, b\right) & =\rho_{a p p}\left(\bar{k}^{*}, 0, \eta, \bar{p}+\bar{q}\left(\bar{k}^{*}\right)^{2}, \bar{p}+\bar{q}\left(\bar{k}^{*}\right)^{2}\right) \\
& =\rho_{a p p}\left(k_{\max }, 0, \eta, \bar{p}+\bar{q} k_{\max }^{2}, \bar{p}+\bar{q} k_{\max }^{2}\right)
\end{aligned}
$$


are given asymptotically by

$$
\bar{p}=2^{\frac{1}{4}} G_{\min }^{\frac{3}{4}} k_{\max }^{\frac{1}{4}}, \quad \bar{q}=2^{-\frac{3}{4}} G_{\min }^{-\frac{1}{4}} k_{\max }^{-\frac{3}{4}},
$$

where $\bar{k}^{*}$ is the unique interior maximum of $\rho_{\text {app }}$ given asymptotically by $\bar{k}^{*}=2^{\frac{1}{2}} G_{\min }^{\frac{1}{2}} k_{\max }^{\frac{1}{2}}$. The above $\bar{p}$ and $\bar{q}$ solve asymptotically the min-max problem (3.13), and the corresponding convergence factor $\rho_{\mathrm{OO} 2}$ satisfies, for $k_{\max } \rightarrow+\infty$, the estimate

$$
\max _{k \in \mathbb{K}}\left|\rho_{O O 2}(k, 0, \eta, \bar{p}, \bar{q}, a, b)\right|=1-2^{\frac{11}{4}} G_{\min }^{\frac{1}{4}} k_{\max }^{-\frac{1}{4}}+O\left(k_{\max }^{-\frac{1}{2}}\right) .
$$

Proof. First, it is easy to show that when $k>0$, the approximate convergence factor $\rho_{a p p}\left(k, 0, \eta, p+q k^{2}, p+q k^{2}\right)$ attains its unique interior maximum at $\bar{k}(p, q)=\sqrt{p / q-2 \eta}$. Let $p:=C_{p} k_{\max }^{\frac{1}{4}}$ and $q:=C_{q} k_{\max }^{-\frac{3}{4}}$. Then $\bar{k}$ behaves like $\sqrt{C_{p} / C_{q}} k_{\max }^{\frac{1}{2}}$ as $k_{\max } \rightarrow \infty$. Inserting these $p$ and $q$ into $\rho_{\mathrm{OO} 2}\left(k_{\min }, 0, \eta, p, q, a, b\right)$ and expanding for $k_{\max }$ large gives

$$
\rho_{O O 2}\left(k_{\min }, 0, \eta, p, q, a, b\right)=1-\frac{8 G_{\min }}{C_{p}} k_{\max }^{-\frac{1}{4}}+O\left(k_{\max }^{-\frac{1}{2}}\right) .
$$

Inserting $\bar{k}=\sqrt{C_{p} / C_{q}} k_{\text {max }}^{\frac{1}{2}}$ and the values of $p$ and $q$ into $\rho_{a p p}\left(k, 0, \eta, p+q k^{2}, p+q k^{2}\right)$ and expanding for $k_{\max }$ large, we obtain

$$
\rho_{\text {app }}\left(\bar{k}, 0, \eta, p+q \bar{k}^{2}, p+q \bar{k}^{2}\right)=1-8 \sqrt{C_{p} C_{q}} k_{\max }^{-\frac{1}{4}}+O\left(k_{\max }^{-\frac{1}{2}}\right) .
$$

Inserting $k=k_{\max }$ and $p$ and $q$ into $\rho_{a p p}\left(k, 0, \eta, p+q k^{2}, p+q k^{2}\right)$ and expanding for $k_{\max }$ large yields

$$
\rho_{a p p}\left(k_{\max }, 0, \eta, p+q k_{\max }^{2}, p+q k_{\max }^{2}\right)=1-\frac{4}{C_{q}} k_{\max }^{-\frac{1}{4}}+O\left(k_{\max }^{-\frac{1}{2}}\right) .
$$

From (3.18), we know that for any $k_{\max }$ large enough (3.20), (3.21), and (3.22) should be equal to each other. We then set the coefficients of the term $k_{\max }^{\frac{1}{4}}$ of these three equations equal to find

$$
\frac{8 G_{\min }}{C_{p}}=\frac{4}{C_{q}}=8 \sqrt{C_{p} C_{q}}
$$

Let $\bar{C}_{p}=2^{\frac{1}{4}} G_{\min }^{\frac{3}{4}}$ and $\bar{C}_{q}=2^{-\frac{3}{4}} G_{\min }^{-\frac{1}{4}}$ denote the solutions to the above equations. Then we get $\bar{p}=\bar{C}_{p} k_{\text {max }}^{\frac{1}{4}}$ and $\bar{q}=\bar{C}_{q} k_{\text {max }}^{\frac{3}{4}}$. In addition, the asymptotic expression of $\bar{k}^{*}$ follows.

For the second result, by inserting the asymptotic expressions of $\bar{p}$ and $\bar{q}$ into $\rho_{O O 2}\left(k_{\min }, 0, \eta, p, q, a, b\right)$ and expanding for $k_{\max }$ large, we obtain

$$
\rho_{O O 2}\left(k_{\min }, 0, \eta, \bar{p}, \bar{q}, a, b\right)=1-2^{\frac{1}{4}} G_{\min }^{\frac{1}{4}} k_{\max }^{-\frac{1}{4}}+O\left(k_{\max }^{-\frac{1}{2}}\right) .
$$

Now we show that $\bar{p}, \bar{q}$ solve the min-max problem (3.13) for $L=0$ asymptotically when $k_{\max }$ becomes large. We rewrite the convergence factor $\rho_{\mathrm{OO} 2}$ as

$$
\rho_{O O 2}(k, 0, \eta, p, q, a, b)=\rho_{b}\left(k, 0, \eta, p+q k^{2}, p+q k^{2}\right) \rho_{a}\left(k, 0, \eta, p+q k^{2}, p+q k^{2}\right),
$$

and denote by $k_{a, b}(0, \bar{p}, \bar{q})$ the solutions of $\rho_{a, b}\left(k_{a, b}, 0, \eta, \bar{p}+\bar{q} k_{a, b}^{2}, \bar{p}+\bar{q} k_{a, b}^{2}\right)=0$. Similar to the proof of Theorem 3.6, we have $k_{a}(0, \bar{p}, \bar{q})<k_{b}(0, \bar{p}, \bar{q})$. In addition, we know 
that both $\rho_{b}\left(k, 0, \eta, \bar{p}+\bar{q} k^{2}, \bar{p}+\bar{q} k^{2}\right)$ and $\rho_{a}\left(k, 0, \eta, \bar{p}+\bar{q} k^{2}, \bar{p}+\bar{q} k^{2}\right)$ are negative and increasing in $k$ for $k \in\left(k_{\min }, k_{a}(0, \bar{p}, \bar{q})\right)$. Then we conclude that, as the product of these two, the convergence factor $\rho_{\mathrm{OO} 2}(k, 0, \eta, \bar{p}, \bar{q}, a, b)$ is positive and decreasing in $k$ for $k \in\left(k_{\min }, k_{a}(0, \bar{p}, \bar{q})\right)$. Thus, $k_{\min }$ is a possible maximum point of $\rho_{O O 2}(k, 0, \eta, \bar{p}, \bar{q}, a, b)$. Note that $k_{a}(0, \bar{p}, \bar{q})$ behaves asymptotically like $\bar{p}=\bar{C}_{p} k_{\text {max }}^{\frac{1}{4}}$. Since it is easy to show that $\rho_{a p p}\left(k, 0, \eta, \bar{p}+\bar{q} k^{2}, \bar{p}+\bar{q} k^{2}\right)$ attains its maxima at $\bar{k}=2^{\frac{1}{2}} G_{\min }^{\frac{1}{2}} k_{\max }^{\frac{1}{2}}$ and $k_{\max }$, by Theorem 3.1, we conclude that $\rho_{\mathrm{OO} 2}(k, 0, \eta, \bar{p}, \bar{q}, a, b)$ asymptotically attains its maxima at $\bar{k}$ and $k_{\max }$ as well. Altogether, $\rho_{\mathrm{OO} 2}(k, 0, \eta, \bar{p}, \bar{q}, a, b)$ has three potential maxima at $k_{\min }, \bar{k}$, and $k_{\max }$ for $k_{\text {max }}$ large enough.

Then, we need to show that if $(p, q) \neq(\bar{p}, \bar{q})$, then there exists a $k^{*}$ at which the convergence factor $\left|\rho_{\mathrm{OO}_{2}}\right|$ is larger than the maximum value in (3.19). Assume that $p:=C_{p} k_{\max }^{\alpha}$ and $q:=C_{q} k_{\max }^{-\beta}, \bar{p}:=\bar{C}_{p} k_{\max }^{\bar{\alpha}}$ and $\bar{q}:=\bar{C}_{q} k_{\max }^{-\bar{\beta}}$ with $\bar{\alpha}=\frac{1}{4}$ and $\bar{\beta}=\frac{3}{4}$. We show first the case $(\alpha, \beta) \neq(\bar{\alpha}, \bar{\beta})$. It suffices to analyze the following cases.

a) $\alpha>\bar{\alpha}, \beta>-\alpha$. In this case, at $k^{*}=k_{\min }$, the convergence factor is

$$
\rho_{O O 2}\left(k^{*}, 0, \eta, p, q, a, b\right)=1-\frac{8 G_{\min }}{C_{p}} k_{\max }^{-\alpha}+o\left(k_{\max }^{-\alpha}\right) .
$$

b) $\alpha>\bar{\alpha}, \beta<-\alpha$. In this case, at $k^{*}=k_{\min }$, the convergence factor is

$$
\left.\rho_{\mathrm{OO} 2}\left(k^{*}, 0, \eta,\right] p, q, a, b\right)=1-\frac{8 G_{\min }}{C_{q} k_{\min }^{2}} k_{\max }^{\beta}+o\left(k_{\max }^{\beta}\right) .
$$

c) $\alpha>\bar{\alpha}, \beta=-\alpha$. In this case, at $k^{*}=k_{\min }$, we have

$$
\rho_{O O 2}\left(k^{*}, 0, \eta, p, q, a, b\right)=1-\frac{8 G_{\min }}{C_{p}+C_{q} k_{\min }^{2}} k_{\max }^{\beta}+o\left(k_{\max }^{\beta}\right) .
$$

d) $\alpha \leq \bar{\alpha}, \beta \geq \bar{\beta}$ but $(\alpha, \beta) \neq(\bar{\alpha}, \bar{\beta})$. In this case, at $k^{*}=C_{k} k_{\max }^{\frac{\alpha+\beta}{2}}$, we have

$$
\rho_{a p p}\left(k^{*}, 0, \eta, p+q\left(k^{*}\right)^{2}, p+q\left(k^{*}\right)^{2}\right)=1-4 \frac{C_{p}}{C_{k}} k_{\max }^{\frac{\alpha-\beta}{2}}+o\left(k_{\max }^{\frac{\alpha-\beta}{2}}\right) .
$$

e) $\alpha \leq \bar{\alpha}, \beta<\bar{\beta}$. In this case, at $k^{*}=k_{\max }$, we have

$$
\rho_{\text {app }}\left(k^{*}, 0, \eta, p+q\left(k^{*}\right)^{2}, p+q\left(k^{*}\right)^{2}\right)=1-\frac{4}{C_{q}} k_{\max }^{\beta-1}+o\left(k_{\max }^{\beta-1}\right) .
$$

For the cases d) and e), we have by Theorem 3.1 that $\rho_{O O 2}\left(k^{*}, 0, \eta, p, q, a, b\right)$ behaves asymptotically the same way as $\rho_{a p p}\left(k^{*}, 0, \eta, p+q\left(k^{*}\right)^{2}, p+q\left(k^{*}\right)^{2}\right)$ for $k_{\max }$ large enough. Then, in each case above we see that at $k^{*}$ the convergence factor $\left|\rho_{O O 2}\left(k^{*}, 0, \eta, p, q, a, b\right)\right|$ behaves like $1-C k_{\max }^{-\delta}$ with $\delta>\frac{1}{4}$.

We then only need to consider the case where $(\alpha, \beta)=(\bar{\alpha}, \bar{\beta})$ but $\left(C_{p}, C_{q}\right) \neq\left(\bar{C}_{p}, \bar{C}_{q}\right)$. From (3.20) we see that if $C_{p}>\bar{C}_{p}$ and $k_{\max }$ is large enough, we have

$$
\left|\rho_{O O 2}\left(k_{\min }, 0, \eta, p, q, a, b\right)\right|>\left|\rho_{O O 2}\left(k_{\min }, 0, \eta, \bar{p}, \bar{q}, a, b\right)\right| .
$$

If $C_{q}>C_{q}^{*}$ and $k_{\max }$ is large enough, then we have from (3.22) together with Theorem 3.1 that

$$
\left|\rho_{\mathrm{OO} 2}\left(k_{\max }, 0, \eta, p, q, a, b\right)\right|>\left|\rho_{O O 2}\left(k_{\max }, 0, \eta, \bar{p}, \bar{q}, a, b\right)\right| .
$$

If $C_{p}<\bar{C}_{p}$ or $C_{q}<\bar{C}_{q}$ and $k_{\max }$ large enough, then, from (3.21) together with Theorem 3.1, we have that

$$
\left|\rho_{O O 2}(\bar{k}, 0, \eta, p, q, a, b)\right|>\left|\rho_{O O 2}(\bar{k}, 0, \eta, \bar{p}, \bar{q}, a, b)\right| .
$$

This ends the proof. 
3.4. Optimized two-sided Robin transmission conditions. In this section we consider the optimized two-sided (O2s for short) Robin transmission condition: $p_{i}>0$ and $q_{i}=0$ for $i=1,2$. We then need to solve the min-max problem

$$
\min _{p_{1}, p_{2}>0}\left(\max _{k \in \mathbb{K}}\left|\rho_{O 2 s}\left(k, L, \eta, p_{1}, p_{2}, a, b\right)\right|\right),
$$

where

$$
\begin{aligned}
\rho_{O 2 s}( & \left.k, L, \eta, p_{1}, p_{2}, a, b\right) \\
= & \frac{\sqrt{\eta+k^{2}} \cosh \left(\sqrt{\eta+k^{2}}(b-L)\right)-p_{1} \sinh \left(\sqrt{\eta+k^{2}}(b-L)\right)}{\sqrt{\eta+k^{2}} \cosh \left(\sqrt{\eta+k^{2}}(a+L)\right)+p_{1} \sinh \left(\sqrt{\eta+k^{2}}(a+L)\right)} \times \\
& \frac{\sqrt{\eta+k^{2}} \cosh \left(\sqrt{\eta+k^{2}} a\right)-p_{2} \sinh \left(\sqrt{\eta+k^{2}} a\right)}{\sqrt{\eta+k^{2}} \cosh \left(\sqrt{\eta+k^{2}} b\right)+p_{2} \sinh \left(\sqrt{\eta+k^{2}} b\right)} .
\end{aligned}
$$

Again, the following results can be obtained using the techniques applied in [20, 23].

THEOREM 3.8 (O2s, overlapping case). For $L>0$, the solutions $p_{1}^{*}$, $p_{2}^{*}$ to

$$
\rho_{O 2 s}\left(k_{\min }, L, \eta, p_{1}^{*}, p_{2}^{*}, a, b\right)=-\rho_{a p p}\left(\hat{k}_{1}^{*}, L, \eta, p_{1}^{*}, p_{2}^{*}\right)=\rho_{a p p}\left(\hat{k}_{2}^{*}, L, \eta, p_{1}^{*}, p_{2}^{*}\right)
$$

are given asymptotically by

$$
p_{1}^{*}=G_{\min }^{\frac{4}{5}} L^{-\frac{1}{5}}, \quad p_{2}^{*}=G_{\min }^{\frac{2}{5}} L^{-\frac{3}{5}},
$$

where $\hat{k}_{1}^{*}=G_{\min }^{\frac{3}{5}} L^{-\frac{2}{5}}$ and $\hat{k}_{2}^{*}=G_{\min }^{\frac{1}{5}} L^{-\frac{4}{5}}$ are the locations of the interior maxima of $\left|\rho_{\text {app }}\right|$. These values of $p_{1}^{*}, p_{2}^{*}$ asymptotically solve the min-max problem (3.23) and the convergence factor $\rho_{O 2 s}$ satisfies, for $L \rightarrow 0$, the estimate

$$
\max _{k \in \mathbb{K}}\left|\rho_{O 2 s}\left(k, L, \eta, p_{1}^{*}, p_{2}^{*}, a, b\right)\right|=1-4 G_{\min }^{\frac{1}{5}} L^{\frac{1}{5}}+O\left(L^{\frac{2}{5}}\right) .
$$

Proof. We first show that the approximate convergence factor $\rho_{a p p}\left(k, L, \eta, p_{1}^{*}, p_{2}^{*}\right)$ attains its interior minimum and maximum asymptotically at $\hat{k}_{1}^{*}=G_{\min }^{\frac{3}{5}} L^{-\frac{2}{5}}$ and $\hat{k}_{2}^{*}=G_{\min }^{\frac{1}{5}} L^{-\frac{4}{5}}$, and then $\rho_{O 2 s}\left(k, L, \eta, p_{1}^{*}, p_{2}^{*}, a, b\right)$ does so as well because of Theorem 3.1. Solving the equation $\partial_{k} \rho_{a p p}\left(k, L, \eta, p_{1}, p_{2}\right)=0$, we get the positive solutions

$$
\begin{aligned}
& \hat{k}_{1,2}\left(L, \eta, p_{1}, p_{2}\right)=\frac{\sqrt{2}}{2} \sqrt{\frac{L p_{1}^{2}+L p_{2}^{2}-2 L \eta+p_{2}+p_{1} \mp \sqrt{\Phi}}{L}} \quad \text { with } \\
& \Phi=p_{2}^{2}+p_{1}^{2}+2 p_{1} p_{2}-2 L^{2} p_{1}^{2} p_{2}^{2}-2 L p_{1}^{2} p_{2}-2 L p_{2}^{2} p_{1}+L^{2} p_{1}^{4}+2 L p_{1}^{3}+L^{2} p_{2}^{4}+2 L p_{2}^{3} .
\end{aligned}
$$

We make the ansatz $p_{1}:=C_{1} L^{-\frac{1}{5}}$ and $p_{2}:=C_{2} L^{-\frac{3}{5}}$. Inserting this ansatz into $\hat{k}_{1,2}$ we get by an expansion in $L$ for $L$ small that $\hat{k}_{1}=\sqrt{C_{1} C_{2}} L^{-\frac{2}{5}}+O(1)$ and $\hat{k}_{2}=\sqrt{C_{2}} L^{-\frac{4}{5}}+O\left(L^{-\frac{2}{5}}\right)$. Using the same ansatz also in $\rho_{O 2 s}\left(k_{\min }, L, \eta, p_{1}, p_{2}, a, b\right)$ and expanding for small $L$ gives

$$
\rho_{O 2 s}\left(k_{\min }, L, \eta, p_{1}, p_{2}, a, b\right)=1-\frac{4 G_{\min }}{C_{1}} L^{\frac{1}{5}}+O\left(L^{\frac{2}{5}}\right) .
$$

Inserting the same ansatz and the expressions for $\hat{k}_{1,2}$ into $-\rho_{a p p}\left(\hat{k}_{1}, L, \eta, p_{1}, p_{2}\right)$ and $\rho_{a p p}\left(\hat{k}_{2}, L, \eta, p_{1}, p_{2}\right)$ and expanding for small $L$, we obtain in addition

$$
\begin{aligned}
-\rho_{a p p}\left(\hat{k}_{1}, L, \eta, p_{1}, p_{2}\right) & =1-4 \sqrt{\frac{C_{1}}{C_{2}}} L^{\frac{1}{5}}+O\left(L^{\frac{2}{5}}\right) \quad \text { and } \\
\rho_{a p p}\left(\hat{k}_{2}, L, \eta, p_{1}, p_{2}\right) & =1-4 \sqrt{C_{2}} L^{\frac{1}{5}}+O\left(L^{\frac{2}{5}}\right) .
\end{aligned}
$$


Since (3.24) should hold for any $L>0$ small, setting the coefficients of the terms $L^{\frac{1}{5}}$ in (3.26), (3.27), and (3.28) equal, we obtain

$$
\frac{4 G_{\min }}{C_{1}}=4 \sqrt{\frac{C_{1}}{C_{2}}}=4 \sqrt{C_{2}} .
$$

Denoting by $C_{1}^{*}=2^{-\frac{8}{5}} \eta^{\frac{2}{5}} G_{\min }^{\frac{4}{5}}$ and $C_{2}^{*}=2^{-\frac{4}{5}} \eta^{\frac{1}{5}} G_{\min }^{\frac{2}{5}}$ the solutions to (3.29), we arrive at the asymptotic expressions of $p_{1,2}^{*}$ and $\hat{k}_{1,2}^{*}$.

We then prove that the parameters $p_{1,2}^{*}$ obtained above solve asymptotically the min-max problem (3.23). We rewrite the convergence factor $\rho_{O 2 s}$ as

$$
\rho_{O 2 s}\left(k, L, \eta, p_{1}, p_{2}, a, b\right)=\rho_{a}\left(k, L, \eta, p_{1}, p_{2}\right) \rho_{b}\left(k, L, \eta, p_{1}, p_{2}\right) .
$$

Then $k_{b}\left(L, p_{1}^{*}\right)$ behaves like $p_{1}^{*}=G_{\min }^{\frac{4}{5}} L^{-\frac{1}{5}}$ and $k_{a}\left(L, p_{2}^{*}\right)$ behaves like $p_{2}^{*}=G_{\min }^{\frac{2}{5}} L^{-\frac{3}{5}}$. By Lemma 3.2 we know that $\rho_{a, b}\left(k, L, \eta, p_{1}^{*}, p_{2}^{*}\right)$ are negative and increasing functions of $k$ for $k \in\left(k_{\min }, k_{b}\left(L, p_{1}^{*}\right)\right)$ and $L$ small enough. Therefore, the convergence factor $\rho_{O 2 s}\left(k, L, \eta, p_{1}^{*}, p_{2}^{*}, a, b\right)$, as the product of $\rho_{a}, \rho_{b}$, decreases in $k$ for $k_{\min } \leq k \leq k_{b}\left(L, p_{1}^{*}\right)$ and $L$ small, hence, $k=k_{\min }$ is a possible maximum point of $\rho_{O 2 s}\left(k, L, \eta, p_{1}^{*}, p_{2}^{*}, a, b\right)$ for $L$ small.

Since $\rho_{a p p}\left(k, L, \eta, p_{1}^{*}, p_{2}^{*}\right)$ attains its unique interior minimum asymptotically at $\hat{k}_{1}$ and attains its unique interior maximum asymptotically at $\hat{k}_{2}$, we know from Theorem 3.1 that when $k>k_{b}\left(L, p_{1}^{*}\right), \rho_{O 2 s}\left(k, L, \eta, p_{1}^{*}, p_{2}^{*}, a, b\right)$ also attains its interior minimum and maximum asymptotically at $\hat{k}_{1}^{*}$ and $\hat{k}_{2}^{*}$, respectively.

We now show that $p_{1,2}^{*}$ given in (3.25) solve the min-max problem (3.23) asymptotically. We make the ansatz $p_{1}:=C_{1} L^{-\alpha}, p_{2}:=C_{2} L^{-\beta}$, and set $p_{1}^{*}:=C_{1}^{*} L^{-\alpha^{*}}, p_{2}^{*}:=C_{2}^{*} L^{-\beta^{*}}$ with $\alpha^{*}=\frac{1}{5}, \beta^{*}=\frac{3}{5}$. Then, we obtain

$$
\rho_{O 2 s}\left(k_{\min }, L, \eta, p_{1}^{*}, p_{2}^{*}, a, b\right)=1-4 G_{\min }^{\frac{1}{5}} L^{\frac{1}{5}}+O\left(L^{\frac{2}{5}}\right) .
$$

We first prove that if $(\alpha, \beta) \neq\left(\alpha^{*}, \beta^{*}\right)$, then there exists a frequency $k^{*}$ such that at this frequency the convergence factor has an asymptotic order larger than $\frac{1}{5}$. We show this by examining in detail the following cases, where we consider the case $\beta \geq \alpha$ only since it is easy to verify that a swapping of the parameters $p_{1}$ and $p_{2}$ will result in the same asymptotic expansions.

a) $\beta \geq \alpha>\alpha^{*}$. In this case, at $k^{*}=k_{\min }$, we have

$$
\begin{aligned}
& \rho_{O 2 s}\left(k^{*}, L, \eta, p_{1}, p_{2}, a, b\right) \\
& \quad=1-\frac{4 G_{\min }}{C_{1}} L^{\alpha}-\frac{4 G_{\min }}{C_{2}} L^{\beta}-4 G_{\min } L+o\left(L^{\min \{\alpha, 1\}}\right) .
\end{aligned}
$$

b) $\alpha<\alpha^{*}, \beta>\beta^{*}$. In this case, at $k^{*}=C_{k} L^{-\frac{2}{5}}$, we have

$$
\begin{aligned}
& -\rho_{\text {app }}\left(k^{*}, L, \eta, p_{1}, p_{2}\right) \\
& \quad=1-2 \frac{C_{1}}{C_{k}} L^{\frac{2}{5}-\alpha}-2 \frac{C_{k}}{C_{2}} L^{\beta-\frac{2}{5}}-2 C_{k} L^{\frac{3}{5}}+o\left(L^{\min \left\{\frac{2}{5}-\alpha, \beta-\frac{2}{5}, \frac{3}{5}\right\}}\right) .
\end{aligned}
$$

c) $\alpha=\alpha^{*}, 1 \geq \beta>\beta^{*}$. In this case, at $k^{*}=C_{k} L^{-\frac{\alpha+\beta}{2}}$, we have

$$
\rho_{a p p}\left(k^{*}, L, \eta, p_{1}, p_{2}\right)=-1+2\left(\frac{C_{1}}{C_{k}}+\frac{C_{k}}{C_{2}}\right) L^{\frac{\beta-\alpha}{2}}+2 C_{k} L^{\frac{2-\alpha-\beta}{2}}+o\left(L^{\frac{\beta-\alpha}{2}}\right) .
$$


d) $\alpha=\alpha^{*}, \beta>1$. In this case, at $k^{*}=C_{k} L^{-\frac{3}{5}}$, we obtain that

$$
\rho_{a p p}\left(k^{*}, L, \eta, p_{1}, p_{2}\right)=-1+2\left(\frac{C_{1}}{C_{k}}+C_{k}\right) L^{\frac{2}{5}}+o\left(L^{\frac{2}{5}}\right) .
$$

e) $\alpha \leq \alpha^{*}, \alpha \leq \beta<\beta^{*}$. In this case, at $k^{*}=C_{k} L^{-\frac{\beta+1}{2}}$, we obtain that

$$
\rho_{a p p}\left(k^{*}, L, \eta, p_{1}, p_{2}\right)=1-2 \frac{C_{1}}{C_{k}} L^{\frac{\beta+1-2 \alpha}{2}}-2\left(\frac{C_{2}}{C_{k}}+C_{k}\right) L^{\frac{1-\beta}{2}}+o\left(L^{\frac{1-\beta}{2}}\right) .
$$

f) $\beta=\beta^{*}, \alpha<\alpha^{*}$. In this case, choosing $k^{*}=C_{k} L^{-\frac{\alpha+\beta}{2}}$ gives

$$
\rho_{\text {app }}\left(k^{*}, L, \eta, p_{1}, p_{2}\right)=-1+2\left(\frac{C_{1}}{C_{k}}+\frac{C_{k}}{C_{2}}\right) L^{\frac{\beta-\alpha}{2}}+o\left(L^{\frac{\beta-\alpha}{2}}\right) .
$$

Note that by Theorem 3.1 in the cases b)-f) we have that $\rho_{O 2 s}\left(k^{*}, L, \eta, p_{1}, p_{2}, a, b\right)$ behaves asymptotically in the same way as $\rho_{a p p}\left(k^{*}, L, \eta, p_{1}, p_{2}\right)$ for $L>0$ small enough. Therefore, in each case above we see that at $k^{*}$ the convergence factor $\left|\rho_{O 2 s}\left(k^{*}, L, R, \eta, p, q\right)\right|$ behaves asymptotically like $1-C L^{\delta}$ with $\delta>\frac{1}{5}$.

We finally consider the case $(\alpha, \beta)=\left(\alpha^{*}, \beta^{*}\right)$ but $\left(C_{1}, C_{2}\right) \neq\left(C_{1}^{*}, C_{2}^{*}\right)$. If $C_{1}>C_{1}^{*}$, then we get from (3.26) that

$$
\left|\rho_{O 2 s}\left(k_{\min }, L, \eta, p_{1}, p_{2}, a, b\right)\right|>\left|\rho_{O 2 s}\left(k_{\min }, L, \eta, p_{1}^{*}, p_{2}^{*}, a, b\right)\right|,
$$

and if $C_{2}<C_{2}^{*}$, we get from (3.28) together with Theorem 3.1 that

$$
\rho_{O 2 s}\left(\hat{k}_{2}, L, \eta, p_{1}, p_{2}, a, b\right)>\rho_{O 2 s}\left(\hat{k}_{2}^{*}, L, \eta, p_{1}^{*}, p_{2}^{*}, a, b\right),
$$

both for $L>0$ small enough. The last case we need to consider is $C_{1}<C_{1}^{*}$ and $C_{2}>C_{2}^{*}$, and we have $\frac{C_{1}}{C_{2}}<\frac{C_{1}^{*}}{C_{2}^{*}}$. Thus from (3.27) together with Theorem 3.1, we have

$$
\left|\rho_{O 2 s}\left(\hat{k}_{1}, L, \eta, p_{1}, p_{2}, a, b\right)\right|>\left|\rho_{O 2 s}\left(\hat{k}_{1}^{*}, L, \eta, p_{1}^{*}, p_{2}^{*}, a, b\right)\right|
$$

for $L>0$ small enough, which concludes the proof.

THEOREM 3.9 (O2s, nonoverlapping case). For vanishing overlap, $L=0$, the solutions $\bar{p}_{1}, \bar{p}_{2}$ to

$$
\rho_{O 2 s}\left(k_{\min }, 0, \eta, \bar{p}_{1}, \bar{p}_{2}, a, b\right)=-\rho_{a p p}\left(\hat{k}^{*}, 0, \eta, \bar{p}_{1}, \bar{p}_{2}\right)=\rho_{a p p}\left(k_{\max }, 0, \eta, \bar{p}_{1}, \bar{p}_{2}\right)
$$

are given asymptotically by

$$
\bar{p}_{1}=2^{\frac{1}{4}} G_{\min }^{\frac{3}{4}} k_{\text {max }}^{\frac{1}{4}}, \quad \bar{p}_{2}=2^{\frac{3}{4}} G_{\min }^{\frac{1}{4}} k_{\text {max }}^{\frac{3}{4}},
$$

where $\hat{k}^{*}=\left(2 G_{\min }\right)^{\frac{1}{2}} k_{\max }^{\frac{1}{2}}$ is the location of the interior maximum of $\left|\rho_{\text {app }}\right|$. The previous $\bar{p}_{1}, \bar{p}_{2}$ asymptotically solve the min-max problem (3.23) and the corresponding convergence factor satisfies, for $k_{\max } \rightarrow \infty$, the estimate

$$
\max _{k \in \mathbb{K}}\left|\rho_{O 2 s}\left(k, 0, \eta, \bar{p}_{1}, \bar{p}_{2}, a, b\right)\right|=1-2^{\frac{7}{4}} G_{\min }^{\frac{1}{4}} k_{\max }^{-\frac{1}{4}}+O\left(k_{\max }^{-\frac{1}{2}}\right) .
$$

Proof. First of all, we solve directly for the root of the derivative of $\rho_{\text {app }}\left(k, 0, \eta, p_{1}, p_{2}\right)$ with respect to $k$ and obtain its unique positive solution as

$$
\hat{k}\left(\eta, p_{1}, p_{2}\right):=\sqrt{p_{1} p_{2}-\eta} .
$$


Let $p_{1}:=C_{1} k_{\max }^{\frac{1}{4}}$ and $p_{2}:=C_{2} k_{\max }^{\frac{3}{4}}$. Inserting these values of $p_{1,2}$ into $\hat{k}$ and expanding for $k_{\text {max }}$ large, we have $\hat{k}=\sqrt{C_{1} C_{2}} k_{\max }^{\frac{1}{2}}+O\left(k_{\max }^{-\frac{1}{2}}\right)$, which, of course, behaves asymptotically like $\hat{k}=\sqrt{C_{1} C_{2}} k_{\max }^{\frac{1}{2}}$. Inserting the values of $p_{1,2}$ into $\rho_{O 2 s}\left(k_{\min }, 0, \eta, p_{1}, p_{2}, a, b\right)$, and expanding for $k_{\max }$ large, we get

$$
\rho_{O 2 s}\left(k_{\min }, 0, \eta, p_{1}, p_{2}, a, b\right)=1-\frac{4 G_{\min }}{C_{1}} k_{\max }^{-\frac{1}{4}}+O\left(k_{\max }^{-\frac{1}{2}}\right) .
$$

Inserting $\hat{k}$ and $p_{1,2}$ into $\rho_{a p p}\left(k, 0, \eta, p_{1}, p_{2}\right)$ and expanding for $k_{\max }$ large, we find

$$
-\rho_{a p p}\left(\hat{k}, 0, \eta, p_{1}, p_{2}\right)=1-4 \sqrt{\frac{C_{1}}{C_{2}}} k_{\max }^{-\frac{1}{4}}+O\left(k_{\max }^{-\frac{1}{2}}\right) .
$$

Moreover, inserting $p_{1,2}$ into $\rho_{a p p}\left(k_{\max }, 0, \eta, p_{1}, p_{2}\right)$ and expanding for $k_{\max }$ large, we obtain

$$
\rho_{\text {app }}\left(k_{\max }, 0, \eta, p_{1}, p_{2}\right)=1-2 C_{2} k_{\max }^{-\frac{1}{4}}+O\left(k_{\max }^{-\frac{1}{2}}\right) .
$$

Since (3.30) holds for any $k_{\max }$ large enough, we set the coefficients of the term $k_{\max }^{-\frac{1}{4}}$ in (3.31)-(3.33) equal to obtain

$$
\frac{4 G_{\min }}{C_{1}}=4 \sqrt{\frac{C_{1}}{C_{2}}}=2 C_{2}
$$

Denoting by $\bar{C}_{1}=2^{\frac{1}{4}} G_{\min }^{\frac{3}{4}}$ and $\bar{C}_{2}=2^{\frac{3}{4}} G_{\min }^{\frac{1}{4}}$ the solutions to the above equations, we obtain the asymptotic expressions of $\bar{p}_{1,2}$ and $\hat{k}^{*}$.

We finally have to prove that the parameters $\bar{p}_{1,2}$ given above asymptotically solve the min-max problem (3.23) for $L=0$ and $k_{\max }$ large enough. Similar techniques as used in Theorem 3.8 show that $\left|\rho_{O 2 s}\left(k, 0, \eta, \bar{p}_{1}, \bar{p}_{2}, a, b\right)\right|$ should attain its maxima at $k_{\min }, \hat{k}_{1}^{*}$, or $k_{\text {max }}$ asymptotically. We then show that if $\left(p_{1}, p_{2}\right) \neq\left(\bar{p}_{1}, \bar{p}_{2}\right)$, then there exists a $k^{*}$ such that

$$
\left|\rho_{O 2 s}\left(k^{*}, 0, \eta, p_{1}, p_{2}, a, b\right)\right|>\left|\rho_{O 2 s}\left(k_{\min }, 0, \eta, p_{1}^{*}, p_{2}^{*}, a, b\right)\right|
$$

for $k_{\max }$ large enough.

Let $p_{1}:=C_{1} k_{\max }^{\alpha}, p_{2}:=C_{2} k_{\max }^{\beta}$, and $\bar{p}_{1}:=\bar{C}_{1} k_{\max }^{\bar{\alpha}}, \bar{p}_{2}:=\bar{C}_{2} k_{\max }^{\bar{\beta}}$ with $(\bar{\alpha}, \bar{\beta})=$ $\left(\frac{1}{4}, \frac{3}{4}\right)$. Similar to Theorem 3.8, we discuss the case $\beta \geq \alpha$ only. It suffices to consider the following cases:

a). $\beta>\alpha>\bar{\alpha}$. In this case, at $k^{*}=k_{\min }$, we have

$$
\left|\rho_{O 2 s}\left(k^{*}, 0, \eta, p_{1}, p_{2}, a, b\right)\right|=1-\frac{4 G_{\min }}{C_{1}} k_{\max }^{-\alpha}+o\left(k_{\max }^{-\alpha}\right) .
$$

b). $\beta=\alpha>\bar{\alpha}$. In this case, at $k^{*}=k_{\min }$, we have

$$
\left|\rho_{O 2 s}\left(k^{*}, 0, \eta, p_{1}, p_{2}, a, b\right)\right|=1-4 G_{\min }\left(\frac{1}{C_{1}}+\frac{1}{C_{2}}\right) k_{\max }^{-\alpha}+o\left(k_{\max }^{-\alpha}\right) .
$$

c). $\alpha \leq \bar{\alpha}, \beta \geq \bar{\beta}$ but $(\alpha, \beta) \neq(\bar{\alpha}, \bar{\beta})$. In this case, at $k^{*}=C_{k} \frac{\frac{\alpha+\beta}{\max }}{\text {, we have }}$

$$
\rho_{a p p}\left(k^{*}, 0, \eta, p_{1}, p_{2}\right)=-1+2\left(\frac{C_{1}}{C_{k}}+\frac{C_{k}}{C_{2}}\right) k_{\max }^{\frac{\alpha-\beta}{2}}+o\left(k_{\max }^{\frac{\alpha-\beta}{2}}\right) .
$$




\section{ETNA}

Kent State University and

Johann Radon Institute (RICAM)

d). $\alpha \leq \bar{\alpha}, \alpha<\beta<\bar{\beta}$. In this case, at $k^{*}=k_{\max }$, we have

$$
\rho_{\text {app }}\left(k^{*}, 0, \eta, p_{1}, p_{2}\right)=1-2 C_{2} k_{\max }^{\beta-1}+o\left(k_{\max }^{\beta-1}\right) .
$$

e). $\beta=\alpha \leq \bar{\alpha}$. In this case, at $k^{*}=k_{\max }$, we have

$$
\rho_{\text {app }}\left(k^{*}, 0, \eta, p_{1}, p_{2}\right)=1-2\left(C_{1}+C_{2}\right) k_{\max }^{\beta-1}+o\left(k_{\max }^{\beta-1}\right) .
$$

Note that in the cases c)-e) we only get the asymptotic expansions of $\rho_{a p p}$ at $k^{*}$. By Theorem 3.1 we know that $\rho_{O 2 s}\left(k^{*}, 0, \eta, p_{1}, p_{2}, a, b\right)$ behaves the same way as $\rho_{a p p}\left(k^{*}, 0, \eta, p_{1}, p_{2}\right)$. Therefore, in each case above, we see that the convergence factor $\left|\rho_{O 2 s}\left(k, 0, \eta, p_{1}, p_{2}, a, b\right)\right|$ behaves at $k^{*}$ asymptotically like $1-C k_{\max }^{-\delta}$ with $\delta>\frac{1}{4}$.

We then consider the case $(\alpha, \beta)=(\bar{\alpha}, \bar{\beta})$ but $\left(C_{1}, C_{2}\right) \neq\left(\bar{C}_{1}, \bar{C}_{2}\right)$. If $C_{1}>\bar{C}_{1}$, then we have from (3.31) that

$$
\left|\rho_{O 2 s}\left(k_{\min }, 0, \eta, p_{1}, p_{2}, a, b\right)\right|>\left|\rho_{O 2 s}\left(k_{\min }, 0, \eta, \bar{p}_{1}, \bar{p}_{2}, a, b\right)\right|
$$

for $k_{\max }$ large enough. If $C_{2}<\bar{C}_{2}$, then we have from (3.33) together with Theorem 3.1 that

$$
\left|\rho_{O 2 s}\left(k_{\max }, 0, \eta, p_{1}, p_{2}, a, b\right)\right|>\left|\rho_{O 2 s}\left(k_{\max }, 0, \eta, \bar{p}_{1}, \bar{p}_{2}, a, b\right)\right|
$$

for $k_{\max }$ large enough. If $C_{1}<\bar{C}_{1}$ and $C_{2}>\bar{C}_{2}$, then we have from (3.32) together with Theorem 3.1 that

$$
\left|\rho_{O 2 s}\left(\bar{k}, 0, \eta, p_{1}, p_{2}, a, b\right)\right|>\left|\rho_{O 2 s}\left(\hat{k}^{*}, 0, \eta, \bar{p}_{1}, \bar{p}_{2}, a, b\right)\right|
$$

for $k_{\max }$ large enough. The second result is obtained by inserting the optimized parameters $\bar{p}_{1,2}$ into (3.31).

4. Discussion. We now discuss how the domain geometry, in our case the subdomain size parameters, affects the performance of the parallel Schwarz algorithm (1.2) and compare the parallel Schwarz methods with the optimized transmission conditions obtained in our analysis to those from the infinite domain decomposition analysis [14].

First of all, we observe that not all positive parameters in the transmission conditions will lead to a convergent Schwarz iteration between the two subdomains, which is similar to the case of a circular domain decomposition; see [20,23]. If we choose for example $p_{1}=100, p_{2}=0.01, q_{1}=q_{2}=0, L=0.01$, then the convergence factor approximately equals $\rho \approx-2.1637$ at $k=\pi$ for the problem parameters $\eta=2, a=0.1, b=0.5$. In fact, note that

$$
\begin{aligned}
& \lim _{p_{1} \rightarrow+\infty} \frac{\sqrt{\eta+k^{2}} \operatorname{coth}\left(\sqrt{\eta+k^{2}}(b-L)\right)-p_{1}}{\sqrt{\eta+k^{2}} \operatorname{coth}\left(\sqrt{\eta+k^{2}}(a+L)\right)+p_{1}}=-1, \\
& \lim _{p_{2} \rightarrow 0} \frac{\sqrt{\eta+k^{2}} \operatorname{coth}\left(\sqrt{\eta+k^{2}} a\right)-p_{2}}{\sqrt{\eta+k^{2}} \operatorname{coth}\left(\sqrt{\eta+k^{2}} b\right)+p_{2}}=\frac{\sqrt{\eta+k^{2}} \operatorname{coth}\left(\sqrt{\eta+k^{2}} a\right)}{\sqrt{\eta+k^{2}} \operatorname{coth}\left(\sqrt{\eta+k^{2}} b\right)}
\end{aligned}
$$

which is approximately $\frac{b}{a}$ for $\sqrt{\eta+k^{2}} \rightarrow 0$, and $\rho_{c l a} \rightarrow 1$ for $\sqrt{\eta+k^{2}} \rightarrow 0$. This shows, together with the fact that the convergence factor is the product of the above three terms, that a large $p_{1}$ and a small $p_{2}$ may lead to a nonconvergent method. This indicates that one should better use the large parameter in the small subdomain and the small parameter in the large subdomain, which is in agreement with the results in [21]. We also see from the above analysis that the larger the difference between the subdomain size is, the larger the measure of the parameter set becomes where the parameters lead to a nonconvergent method. 
Next, we show that the finiteness and asymmetry of the domain decomposition influences the performance of the parallel Schwarz algorithm (1.2) with the various transmission conditions. We investigate first some properties of the geometry function $G$ described in Lemma 2.2. Since $\operatorname{coth}(x)>1$, for $x>0$, we have $G(k, \eta, a, b)>\sqrt{\eta+k^{2}}=: \bar{G}$ for all $k>0$. In addition, it is easy to show that $G(k, \eta, a, b)$ decreases monotonically to $\bar{G}$ as $a, b \rightarrow \infty$. Therefore, when $a, b \rightarrow \infty$ we have $\lim _{a, b \rightarrow \infty} G(k, \eta, a, b)=\bar{G}$, and the optimized transmission conditions in our analysis converge to their counterparts obtained by the infinite domain decomposition analysis [14]. This result can also be obtained by directly comparing the corresponding transmission conditions found in both cases. Note that the resulting maxima of the convergence factor are given by $\max _{k}|\rho|=1-C\left(G_{\min } L\right)^{\delta}$ for the overlapping case with $\delta=1,1 / 2,1 / 3,1 / 5$ and $\max _{k}|\rho|=1-C\left(G_{\min } / k_{\max }\right)^{\delta}$ for the nonoverlapping case with $\delta=1,1 / 2,1 / 4$. Thus, we conclude that the transmission conditions obtained in our analysis perform better than those obtained from the infinite domain decomposition analysis. Moreover, from the fact that $\left(\left(\operatorname{coth}\left(\sqrt{\eta+k^{2}} a\right)+\operatorname{coth}\left(\sqrt{\eta+k^{2}} b\right)\right) / 2\right)^{\gamma}$ decreases monotonically to 1 as $\gamma \rightarrow 0$, we conclude that the subdomain parameters have more influence on the optimized Schwarz methods with $\delta$ large than those with $\delta$ small. Note that the subdomain parameters $a, b$ enter the function $G$ (defined in Lemma 2.2) as exponential powers; the influence increases exponentially in the subdomain size parameters when they tend to zero, which shows that if a subdomain size is very small, then one has to take this into account in the transmission conditions.

Next, we show that balancing the domain decomposition provides a benefit for the optimized Schwarz method. To this end, we consider $\Omega=\{(x, y) \mid x \in(-c, c), y \in(-d, d)\}$ a rectangular domain, and assume $c>d$, which means the domain is a flat strip. We have two ways of decomposing the domain: choosing the interface vertically to be at $x=0$, the corresponding function $G$ is given by $G_{v}=\frac{1}{4} \sqrt{\frac{\pi^{2}}{d^{2}}+4 \eta} /\left(1-\exp \left(-2 c \sqrt{\frac{\pi^{2}}{d^{2}}+4 \eta}\right)\right)$. Choosing the interface horizontally at $y=0$, which means making even two flatter stripshaped subdomains, $G$ is given by $G_{h}=\frac{1}{4} \sqrt{\frac{\pi^{2}}{c^{2}}+4 \eta} /\left(1-\exp \left(-2 d \sqrt{\frac{\pi^{2}}{c^{2}}+4 \eta}\right)\right)$. Since $c>d$, we have $G_{v}>G_{h}$. Our asymptotic convergence factor estimates then show that decomposing a flat strip into even flatter strips is not a good idea for all the Schwarz methods we considered here; it is better to cut the strip into two shorter, more square-shaped pieces.

Finally, we note that the asymmetry of the domain decomposition can have more influence on the optimized two-sided Robin transmission conditions. In [21] we have shown that in the large subdomain using the small parameter and in the small subdomain using the large parameter can improve the performance of the optimized two-sided Robin transmission condition compared to the reverse order.

5. Numerical examples. We now present some numerical examples to illustrate our analysis. We consider the model problem

$$
\begin{aligned}
(\triangle-2) u & =f, \quad \text { in } \Omega \\
\left.u\right|_{\partial \Omega} & =0
\end{aligned}
$$

with $\Omega=(-a, b) \times(0,1)$ decomposed into $\Omega_{1}=(-a, L) \times(0,1)$ and $\Omega_{2}=(0, b) \times(0,1)$, $b=0.5$. We discretize (5.1) with the classical five-point finite difference scheme on a uniform mesh with mesh parameter $h$ and simulate directly the error equations, i.e., $f=0$. The initial vector of the interface is chosen randomly so that all frequencies are present. We count the number of iterations required to reach a relative error reduction of $1 \mathrm{e}-6$ and compare the results obtained with our parameters to those obtained with parameters from the infinite domain decomposition analysis (see [14]) denoted by the subscript "Inf". We present in Table 5.1, for the overlap $L=h$, the number of iterations required by the Schwarz method 


\section{ETNA}

Kent State University and

Johann Radon Institute (RICAM)

TABLE 5.1

Number of iterations required by the Schwarz algorithms with different transmission conditions with an overlap of one mesh size and varying domain size parameter a.

\begin{tabular}{l|ccc|ccc|ccc|ccc}
$\mathrm{h}$ & \multicolumn{3}{|c|}{$1 / 100$} & \multicolumn{3}{|c|}{$1 / 200$} & \multicolumn{3}{|c}{$1 / 400$} & \multicolumn{3}{c}{$1 / 800$} \\
$a$ & 0.05 & 0.1 & 0.2 & 0.05 & 0.1 & 0.2 & 0.05 & 0.1 & 0.2 & 0.05 & 0.1 & 0.2 \\
\hline \hline Classical & 52 & 78 & 104 & 101 & 153 & 205 & 198 & 304 & 407 & 392 & 605 & 816 \\
\hline $\mathrm{T} 0$ & 9 & 11 & 14 & 11 & 15 & 19 & 16 & 21 & 26 & 23 & 29 & 38 \\
$\mathrm{~T} 0$ & 23 & 22 & 21 & 33 & 30 & 31 & 45 & 44 & 43 & 62 & 61 & 61 \\
\hline $\mathrm{OO} 0$ & 7 & 8 & 9 & 8 & 9 & 11 & 10 & 12 & 14 & 12 & 15 & 17 \\
$\mathrm{OO} 0_{\text {Inf }}$ & 10 & 10 & 10 & 12 & 12 & 12 & 15 & 15 & 15 & 18 & 18 & 18 \\
\hline $\mathrm{OO} 2$ & 8 & 5 & 5 & 7 & 5 & 6 & 6 & 6 & 6 & 6 & 7 & 7 \\
$\mathrm{OO}_{\text {Inf }}$ & 6 & 5 & 5 & 7 & 6 & 6 & 8 & 7 & 7 & 9 & 8 & 8 \\
\hline $\mathrm{O} 2 \mathrm{~s}$ & 6 & 7 & 8 & 8 & 8 & 9 & 10 & 10 & 11 & 10 & 12 & 13 \\
$\mathrm{O}^{2} s_{\text {Inf }}$ & 8 & 8 & 8 & 9 & 9 & 10 & 11 & 11 & 11 & 13 & 13 & 13
\end{tabular}

TABLE 5.2

Number of iterations required by the Schwarz algorithms with different transmission conditions without overlap and varying domain size parameter $a$.

\begin{tabular}{l|ccc|ccc|ccc|ccc}
$\mathrm{h}$ & \multicolumn{3}{|c|}{$1 / 100$} & \multicolumn{3}{|c|}{$1 / 200$} & \multicolumn{3}{c|}{$1 / 400$} & \multicolumn{3}{c}{$1 / 800$} \\
$a$ & 0.05 & 0.1 & 0.2 & 0.05 & 0.1 & 0.2 & 0.05 & 0.1 & 0.2 & 0.05 & 0.1 & 0.2 \\
\hline \hline $\mathrm{T} 0$ & 81 & 142 & 241 & 158 & 287 & 466 & 321 & 579 & 953 & 640 & 1146 & 1926 \\
$\mathrm{~T}_{\text {Inf }}$ & 626 & 612 & 620 & 1265 & 1298 & 1260 & 2526 & 2541 & 2538 & 5098 & 5084 & 5127 \\
\hline $\mathrm{OO} 0$ & 17 & 23 & 29 & 25 & 33 & 40 & 36 & 46 & 55 & 49 & 64 & 76 \\
$\mathrm{OO} 0_{\text {Inf }}$ & 28 & 28 & 28 & 40 & 39 & 39 & 55 & 55 & 56 & 77 & 78 & 79 \\
\hline $\mathrm{OO} 2$ & 6 & 7 & 8 & 7 & 8 & 9 & 9 & 10 & 11 & 10 & 12 & 13 \\
$\mathrm{OO} 2_{\text {Inf }}$ & 8 & 8 & 8 & 10 & 10 & 10 & 12 & 12 & 12 & 14 & 14 & 14 \\
\hline $\mathrm{O} 2 \mathrm{~s}$ & 14 & 14 & 15 & 16 & 17 & 18 & 18 & 19 & 21 & 23 & 24 & 26 \\
$\mathrm{O} s_{\text {Inf }}$ & 15 & 15 & 15 & 18 & 18 & 18 & 23 & 23 & 23 & 27 & 27 & 27
\end{tabular}

with transmission conditions from our analysis compared to those from the infinite domain decomposition analysis with the subdomain size parameter $a$ varying from 0.05 to 0.2 . The same comparison for the nonoverlapping case is given in Table 5.2. We observe that these results illustrate our analysis well. The only exception is the second-order transmission condition with overlap and $a=0.05$, where we would need a more refined mesh to reach the asymptotic regime. We see that our transmission conditions perform better than those from the infinite domain decomposition analysis, especially when the subdomain parameter $a$ is small and the decomposition is very far from being symmetric.

Since the convergence factor estimate of each Schwarz algorithm is of the form $\max _{k}|\rho|=1-C\left(G_{\min } L\right)^{\delta}$ for the overlapping case with $\delta=1,1 / 2,1 / 3,1 / 5$ and $\max _{k}|\rho|=1-C\left(G_{\min } / k_{\max }\right)^{\delta}$ for the nonoverlapping case with $\delta=1,1 / 2,1 / 4$, we also observe from the numerical results that the subdomain size influences the performance more for large values of $\delta$. For example, when the subdomain size parameter is doubled, the number of iterations required by the classical Schwarz method increases by a half, whereas for the optimized transmission condition of order 2 , the number of iterations required increases very slow when increasing the subdomain size parameter $a$.

We present the above results also in loglog plots in Figure 5.1. We see that all the transmission conditions follow the predicted asymptotic convergence order very well except for the optimized two-sided Robin transmission conditions for the case with overlap obtained 

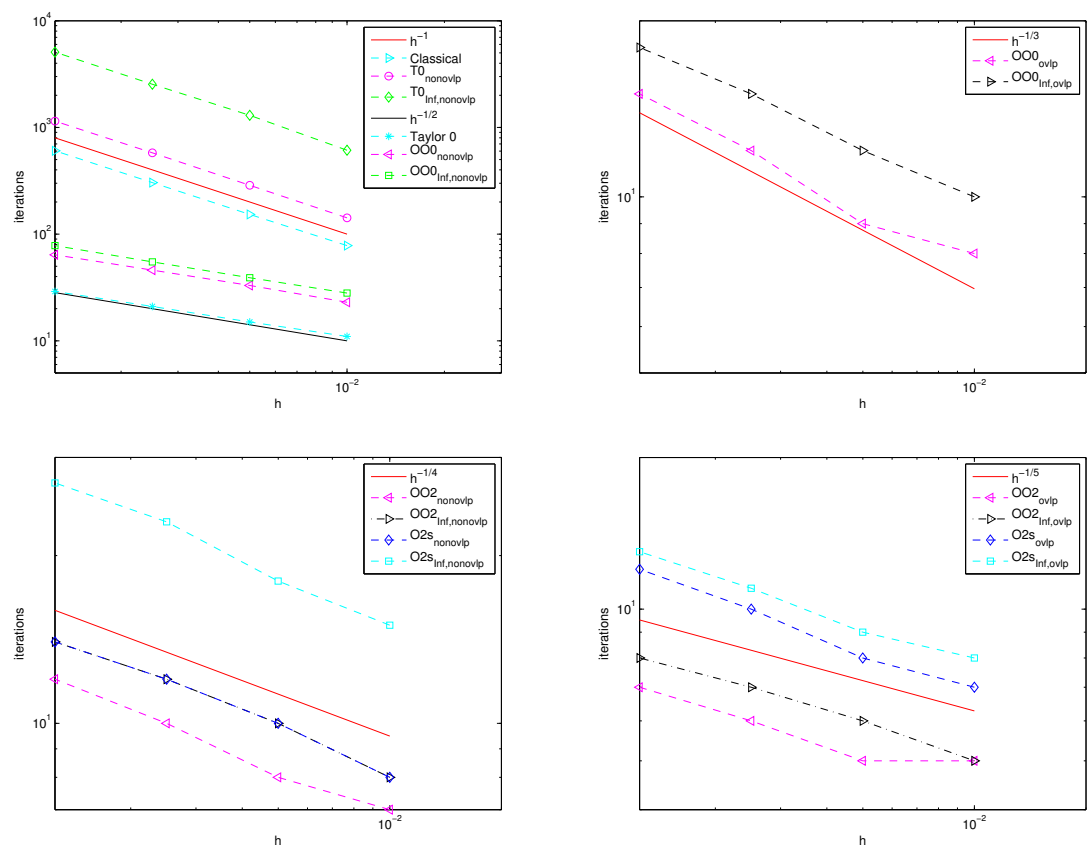

FIG. 5.1. Loglog plots of iteration numbers required by the Schwarz algorithms with transmission conditions from our analysis compared to those from the infinite domain decomposition analysis subscripted by "Inf". The subscript "ovlp" indicates the transmission condition from the overlapping domain decomposition, while "nonovlp" means those from the nonoverlapping one.

from both our analysis and the infinite domain decomposition analysis. Here an even finer mesh would be required, but this problem can be solved by swapping the two parameters directly; see [21] for more details.

We next investigate how well the continuous analysis predicts the optimal parameters to be used in the numerical setting. To this end, we vary the parameter $p$ in the Robin transmission conditions for a fixed problem of mesh size $h=1 / 200$ and count for each value of $p$ the number of iterations to reach a residual of $1 \mathrm{e}-6$. For the optimized transmission condition of order 2, we similarly vary the parameter pair $(p, q)$. The results are given in Figure 5.2 for the overlapping case and in Figure 5.3 for the nonoverlapping case. These results show that the analysis very well predicts the optimal parameter especially for the overlapping case. For the optimized two-sided transmission conditions, we refer to [21].

In each plot, we also compare the optimized parameters from our analysis to those from the infinite domain decomposition analysis (see [14]), which are indicated by "o". We see that our optimized parameters perform better than those from the infinite domain decomposition analysis.

Finally, we would like to test the case where both geometric parameters $a$ and $b$ are very small. The number of iterations required by different transmission parameters for $a=b=0.01$ are reported in Table 5.3, where we see that for each transmission condition, our new optimized parameters involving the subdomain geometry leads to much faster algorithms for both overlapping and nonoverlapping cases than those from the infinite domain decomposition analysis. Noting that a Krylov acceleration generally improves the performance by about 


\section{ETNA}

Kent State University and

Johann Radon Institute (RICAM)
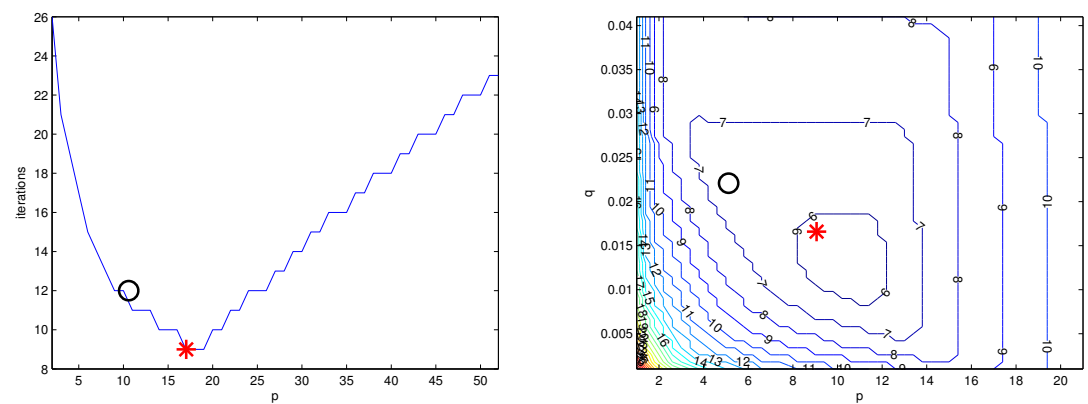

FIG. 5.2. With overlap of one mesh size $h$, the optimized parameter $(*)$ found by the analytical optimization compared to the optimized parameter ( $(0)$ found by the infinite domain decomposition analysis reported in [14] and to the performance of other values of the parameters: left for the Robin case, right for the second-order case.
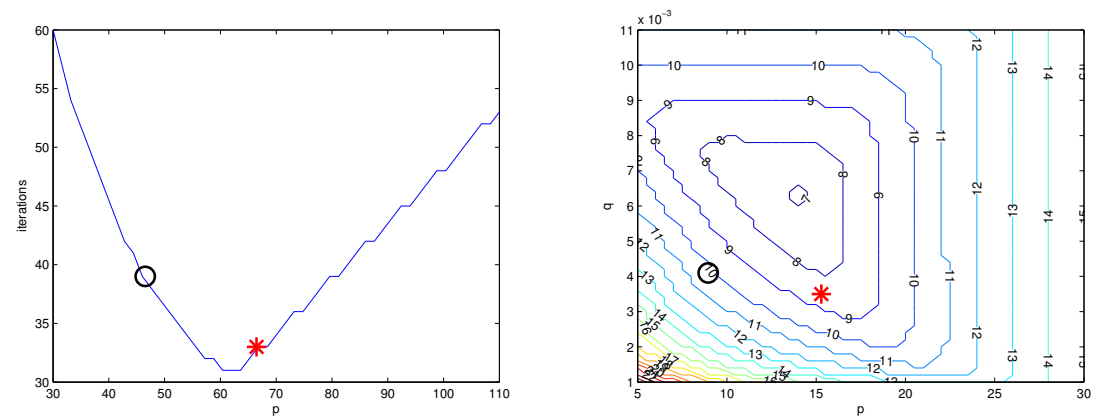

FIG. 5.3. Without overlap, the optimized parameter $(*)$ found by the analytical optimization compared to the optimized parameter ( 0 ) found by the infinite domain decomposition analysis reported in [14] and to the performance of other values of the parameters: left for the Robin case, right for the second-order case.

TABLE 5.3

Number of iterations required by optimized Schwarz algorithms with overlap $h$ and without overlap (in parentheses) for the domain parameters $a=b=0.01$.

\begin{tabular}{l|c|c|c|c}
$\mathrm{h}$ & $1 / 1000$ & $1 / 2000$ & $1 / 4000$ & $1 / 8000$ \\
\hline \hline $\mathrm{OO} 0$ & $7(20)$ & $9(28)$ & $11(39)$ & $13(54)$ \\
$\mathrm{OOO} 0_{\text {Inf }}$ & $22(88)$ & $25(124)$ & $31(177)$ & $39(247)$ \\
\hline $\mathrm{OO} 2$ & $5(7)$ & $5(8)$ & $6(9)$ & $6(11)$ \\
$\mathrm{OO} 2_{\text {Inf }}$ & $46(26)$ & $41(22)$ & $39(22)$ & $35(25)$ \\
\hline $\mathrm{O} 2 \mathrm{~s}$ & $7(12)$ & $8(14)$ & $9(17)$ & $11(20)$ \\
$\mathrm{O}^{2} s_{\text {Inf }}$ & $13(29)$ & $15(35)$ & $18(41)$ & $20(49)$
\end{tabular}

a square root (see [14]), we can expect that our new optimized parameters are much more efficient than those from the infinite domain analysis in applications.

6. Conclusion. We analyzed optimized Schwarz methods for a model problem with finite two-subdomain decomposition and investigated the influence of a domain truncation on their performance. Similar to the circular domain decomposition, an approximate convergence factor has to be introduced to accomplish this analysis. We found that not all positive transmission parameters result in convergent Schwarz methods for asymmetric domain decom- 
positions. This result is similar to the circular domain decomposition case, but differs from the infinite domain decomposition case, where the Schwarz methods converge for all positive transmission parameters. We derived closed-form asymptotic formulas for the optimized transmission parameters and found that the subdomain size parameters $a$ and $b$ enter the optimized transmission parameters through a geometry function $G(k, \eta, a, b)$. The asymptotic convergence factors satisfy the estimates $\max _{k}|\rho|=1-C\left(G_{\min } L\right)^{\delta}$ for the overlapping case when the overlap $L$ is small, with $\delta=1,1 / 2,1 / 3,1 / 5$, and $\max _{k}|\rho|=1-C\left(G_{\min } / k_{\max }\right)^{\delta}$ for the nonoverlapping case when $k_{\max }$ is large, with $\delta=1,1 / 2,1 / 4$. They perform much better than those obtained from the infinite domain decomposition analysis especially for the cases where the subdomains are very narrow strips. We also found that the subdomain size parameters have more influence on the performance of the optimized Schwarz methods with large $\delta$ than those with small $\delta$. Finally, our analysis revealed for a two-subdomain decomposition that one should choose a decomposition such that subdomains are closer to squares than to flat strips for good performance.

\section{REFERENCES}

[1] A. Alonso RodrígueZ AND L. Gerardo-Giorda, New nonoverlapping domain decomposition methods for the harmonic Maxwell system, SIAM J. Sci. Comput., 28 (2006), pp. 102-122.

[2] E. Audusse, P. DReyfuss, AND B. Merlet, Optimized Schwarz waveform relaxation for the primitive equations of the ocean, SIAM J. Sci. Comput., 32 (2010), pp. 2908-2936.

[3] J.-D. BenAMOU AND B. DeSPRÈs, A domain decomposition method for the Helmholtz equation and related optimal control problems, J. Comput. Phys., 136 (1997), pp. 68-82.

[4] D. Bennequin, M. J. GANDER, AND L. HALPERn, A homographic best approximation problem with application to optimized Schwarz waveform relaxation, Math. Comp., 78 (2009), pp. 185-223.

[5] Z. CHEN, M. J. GANDER, AND H. ZHANG, On the relation between optimized Schwarz methods and source transfer, in Domain Decomposition Methods in Science and Engineering XXII, T. Dickopf, M. J. Gander, L. Halpern, R. Krause, and L. F. Pavarino, eds., vol. 104 of Lect. Notes Comput. Sci. Eng., Springer, Cham, 2016, pp. 217-225.

[6] Z. Chen AND X. XIANG, A source transfer domain decomposition method for Helmholtz equations in unbounded domain, SIAM J. Numer. Anal., 51 (2013), pp. 2331-2356.

[7] B. Després, Décomposition de domaine et problème de Helmholtz, C. R. Acad. Sci. Paris Sér. I Math., 311 (1990), pp. 313-316.

[8] C. R. DohrmanN, A preconditioner for substructuring based on constrained energy minimization, SIAM J. Sci. Comput., 25 (2003), pp. 246-258.

[9] V. Dolean, M. J. Gander, And L. Gerardo-Giorda, Optimized Schwarz methods for Maxwell's equations, SIAM J. Sci. Comput., 31 (2009), pp. 2193-2213.

[10] V. Dolean, P. Jolivet, AND F. NataF, An Introduction to Domain Decomposition Methods: Algorithms, Theory, and Parallel Implementation, SIAM, Philadelphia, 2015.

[11] M. El Bouajaji, V. Dolean, M. J. Gander, AND S. LANTERI, Optimized Schwarz methods for the time-harmonic Maxwell equations with damping, SIAM J. Sci. Comput., 34 (2012), pp. A2048-A2071.

[12] B. ENGQUIST AND L. YING, Sweeping preconditioner for the Helmholtz equation: moving perfectly matched layers, Multiscale Model. Simul., 9 (2011), pp. 686-710.

[13] C. FARHAT AND F.-X. ROUX, A method of finite element tearing and interconnecting and its parallel solution algorithm, Internat. J. Numer. Methods Engrg., 32 (1991), pp. 1205-1227.

[14] M. J. GANDER, Optimized Schwarz methods, SIAM J. Numer. Anal., 44 (2006), pp. 699-731.

[15] - On the influence of geometry on optimized Schwarz methods, SeMA J., (2011), pp. 71-78.

[16] M. J. GANDER AND L. HALPERN, Optimized Schwarz waveform relaxation methods for advection reaction diffusion problems, SIAM J. Numer. Anal., 45 (2007), pp. 666-697.

[17] M. J. GANDER AND F. KWOK, Best Robin parameters for optimized Schwarz methods at cross points, SIAM J. Sci. Comput., 34 (2012), pp. A1849-A1879.

[18] M. J. GANDER, S. LoISEL, AND D. B. SZYLD, An optimal block iterative method and preconditioner for banded matrices with applications to PDEs on irregular domains, SIAM J. Matrix Anal. Appl., 33 (2012), pp. 653-680.

[19] M. J. GANDER, F. MAGOULÈS, AND F. NATAF, Optimized Schwarz methods without overlap for the Helmholtz equation, SIAM J. Sci. Comput., 24 (2002), pp. 38-60.

[20] M. J. GANDER AND Y. XU, Optimized Schwarz methods for circular domain decompositions with overlap, SIAM J. Numer. Anal., 52 (2014), pp. 1981-2004. 
[21] _ Optimized Schwarz method with two-sided transmission conditions in an unsymmetric domain decomposition, in Domain Decomposition Methods in Science and Engineering XXII, T. Dickopf, M. J. Gander, L. Halpern, R. Krause, and L. F. Pavarino, eds., vol. 104 of Lect. Notes Comput. Sci. Eng., Springer, Cham, 2016, pp. 631-639.

[22] _ Optimized Schwarz methods for model problems with continuously variable coefficients, SIAM J. Sci. Comput., 38 (2016), pp. A2964-A2986.

[23] _ Optimized Schwarz methods with nonoverlapping circular domain decomposition, Math. Comp., 86 (2017), pp. 637-660.

[24] M. J. GANDER AND H. ZHANG, Optimized Schwarz methods with overlap for the Helmholtz equation, in Domain Decomposition Methods in Science and Engineering XXI, J. Erhel, M. J. Gander, L. Halpern, G. Pichot, T. Sassi, and O. Widlund, eds., vol. 98 of Lect. Notes Comput. Sci. Eng., Springer, Cham, 2014, pp. 207-215.

[25] J. C. Garay, F. Magoulès, AND D. B. Szyld, Optimized Schwarz method for Poisson's equation in rectangular domains, in Domain Decomposition Methods in Science and Engineering XXIV, P. E. Bjørstad, S. C. Brenner, L. Halpern, R. Kornhuber, H. J. Kim, T. Rahman, O. B. Widlund, eds., vol. 125 of Lect. Notes Comput. Sci. Eng., Springer, Cham, 2018, in press.

[26] _ - Synchronous and asynchronous optimized Schwarz method for Poisson's equation in rectangular domains, Tech. Report 17-10-18, Department of Mathematics, Temple University, Philadelphia, 2017.

[27] L. Gerardo-Giorda, F. Nobile, AND C. Vergara, Analysis and optimization of Robin-Robin partitioned procedures in fluid-structure interaction problems, SIAM J. Numer. Anal., 48 (2010), pp. 2091-2116.

[28] L. GerARDO-GIORDA AND M. PEREGO, Optimized Schwarz methods for the bidomain system in electrocardiology, ESAIM Math. Model. Numer. Anal., 47 (2013), pp. 583-608.

[29] J.-H. KIMN, A convergence theory for an overlapping Schwarz algorithm using discontinuous iterates, Numer. Math., 100 (2005), pp. 117-139.

[30] L. LAAYOUNI AND D. B. SZYLD, On the performance of the algebraic optimized Schwarz methods with applications, Numer. Algorithms, 67 (2014), pp. 889-916.

[31] P.-L. LIONS, On the Schwarz alternating method. III. A variant for nonoverlapping subdomains, in Third International Symposium on Domain Decomposition Methods for Partial Differential Equations, T. Chan, R. Glowinski, J. Periaux, and O. Widlund, eds., SIAM, Philadelphia, 1990, pp. 202-223.

[32] S. LOISEL, Condition number estimates for the nonoverlapping optimized Schwarz method and the 2-Lagrange multiplier method for general domains and cross points, SIAM J. Numer. Anal., 51 (2013), pp. 3062-3083.

[33] S. LOISEL AND D. B. SZYLD, On the geometric convergence of optimized Schwarz methods with applications to elliptic problems, Numer. Math., 114 (2010), pp. 697-728.

[34] V. MARTIN, An optimized Schwarz waveform relaxation method for the unsteady convection diffusion equation in two dimensions, Appl. Numer. Math., 52 (2005), pp. 401-428.

[35] F. NATAF, Optimized Schwarz methods, in Domain Decomposition Methods in Science and Engineering XVIII, M. Bercovier, M. J. Gander, R. Kornhuber, and O. B. Widlund, eds., vol. 70 of Lect. Notes Comput. Sci. Eng., Springer, Berlin, 2009, pp. 233-240.

[36] Z. PENG AND J.-F. LEE, Non-conformal domain decomposition method with second-order transmission conditions for time-harmonic electromagnetics, J. Comput. Phys., 229 (2010), pp. 5615-5629.

[37] Z. PENG, V. RAWAT, AND J.-F. LEE, One way domain decomposition method with second order transmission conditions for solving electromagnetic wave problems, J. Comput. Phys., 229 (2010), pp. 1181-1197.

[38] J. Poulson, B. ENGQuist, S. LI, AND L. YING, A parallel sweeping preconditioner for heterogeneous 3D Helmholtz equations, SIAM J. Sci. Comput., 35 (2013), pp. C194-C212.

[39] A. QADDOURI, Optimized Schwarz methods with the Yin-Yang grid for shallow water equations, in Domain Decomposition Methods in Science and Engineering XVII, U. Langer, M. Discacciati, D. E. Keyes, O. B. Widlund, and W. Zulehner, eds., vol. 60 of Lect. Notes Comput. Sci. Eng., Springer, Berlin, 2008, pp. 347-353.

[40] A. QAdDouri, L. LAayouni, S. Loisel, J. CôTÉ, AND M. J. GANDER, Optimized Schwarz methods with an overset grid for the shallow-water equations: preliminary results, Appl. Numer. Math., 58 (2008), pp. 459-471.

[41] L. QIN AND X. XU, Optimized Schwarz methods with Robin transmission conditions for parabolic problems, SIAM J. Sci. Comput., 31 (2008), pp. 608-623.

[42] A. Toselli AND O. Widlund, Domain Decomposition Methods-Algorithms and Theory, Springer, Berlin, 2005.

[43] P. Tsuji, B. ENGQuist, AND L. YING, A sweeping preconditioner for time-harmonic Maxwell's equations with finite elements, J. Comput. Phys., 231 (2012), pp. 3770-3783.

[44] P. TsujI AND L. YING, A sweeping preconditioner for Yee's finite difference approximation of time-harmonic Maxwell's equations, Front. Math. China, 7 (2012), pp. 347-363.

[45] A. Vion And C. GeuZAine, Double sweep preconditioner for optimized Schwarz methods applied to the Helmholtz problem, J. Comput. Phys., 266 (2014), pp. 171-190. 